\title{
LAS ASTERÁCEAS DE LA CUENCA DEL RÍO CHILLÓN (CANTA, LIMA, PERÚ)
}

\section{THE ASTERACEAE OF THE CHILLON RIVER BASIN (CANTA, LIMA, PERU)}

\author{
Graciela Vilcapoma ${ }^{1}$ y Hamiltón Beltrán ${ }^{2}$
}

\begin{abstract}
Resumen
Se presenta una lista de 235 especies de Asteráceas nativas y/o naturalizadas que corresponden a 12 tribus y 95 géneros de la cuenca del rio Chillón; además, para las especies, se proporciona su rango altitudinal asi como la ocurrencia en otros departamentos del Perú. Las especies reportadas estan respaldadadas por material de herbario. Diez especies son adicionadas como nuevos registros para la cuenca del Chillón.

Palabras clave: compositae, Andes occidentales, diversidad, endemismo.
\end{abstract}

\begin{abstract}
A list of 235 native and/or naturalized Asteraceae species corresponding to 12 tribes and 95 genera of the Chillon River Basin is presented. In addition, each species is provided with its altitudinal range as well as occurrence in other departments of Peru.The reported species are supported by herbarium material. Ten species are added as new records for the Chillon basin.
\end{abstract}

Key words: composite, western slopes of the Andes, diversity, endemic species.

\section{Introducción.}

Perú está considerado como zona de alta riqueza de plantas y principalmente de las Asteráceas, con más de 1613 especies (Ulloa et al., 2017). Para la región Lima están documentadas 306 especies (Gonzáles, 2016), de éstas 63 son endémicas nacionales (Beltrán et al., 2006). Las Asteráceas están concentradas en las vertientes occidentales y orientales entre $3900 \mathrm{msnm}$ y el límite de la vegetación donde alcanza su máxima abundancia (Ball, 1885; Smith, 1989; Yarupaitán, 2003; Cano et al., 2010, 2011), en los bosques amazónicos esta familia está pobremente representada (Spichiger et al., 2002; Castillo, 2006). Beltrán et al. (2006) reconocen 724 especies endémicas para el pais, de las cuales Senecio (97 spp.), Gynoxys (45 spp.) y Verbesina (44 spp.) tienen el mayor número de especies. Para la región Lima son escasos los estudios de Asteráceas (Meza, 1966; De la Cruz, 1999; Beltrán, 2016; Gonzáles, 2016).

El objetivo de este trabajo es dar a conocer las especies de la familia de Asteráceas de la cuenca del río Chillón, cuyas especies se encuentran distribuidas desde el nivel del mar hasta los 4800 msnm, concentrándose el mayor número de especies entre los 3000 y $4000 \mathrm{msnm}$.

La cuenca del río Chillón fue visitada por varias expediciones científicas, durante los años 1778 a 1925.

Estas colecciones están conservadas en; el Real Jardín botánico de Madrid (MA) colectadas en Canta y Obrajillo por H. Ruiz y J. Pavón (1777-1778), también las de L. Nee y T. Haenke (1789) que visitaron Puruchuco, Huamantanga, San Buenaventura, Canta y Obrajillo; Royal Botanic de Londres (K) las de A.
Cruckshank (1829), recolectadas en San Buenaventura y Huamantanga, además de A. Mathew (1833) quien recolectó en Obrajillo, Huamantanga y Puruchuco; Smithsonian Institution de Washington (US) conserva las colecciones de J. D. Brackenridge, Ch. Pickering y W. Rich integrantes de la expedición Wilkes (18381842) quienes recolectaron en Yangas, Obrajillo y Cullhuay; Field Museum of Chicago (F) conserva las colecciones de F. W. Pennell (1925), recogidas en Santa Rosa de Quives, San Buenaventura y Obrajillo. Área de estudio.

La cuenca del río Chillón (Figura 1) está comprendida entre los $11^{\circ} 20^{\prime}-12^{\circ} 00^{\prime}$ de latitud Sur y $\operatorname{los} 76^{\circ} 20^{\prime}-77^{\circ} 10^{\prime}$ longitud Oeste, ocupa territorios de las Provincias de Lima y Canta, siendo esta última la de mayor superficie. Está irrigada por el río del mismo nombre, que tiene sus nacientes en los nevados y lagunas de Chontas a $4800 \mathrm{msnm}$, en su primer recorrido recibe aguas de los nevados y lagunas de la cordillera La Viuda, principalmente de la laguna de Chuchún a $4400 \mathrm{msnm}$. Después de recorrer 146 kilómetros desemboca en el Océano Pacífico al norte de la ciudad de Lima (ONERN, 1975; Rivera, 1981). Las variaciones orográficas de la cuenca determinan diferencias climáticas notorias entre la cuenca baja (templado) y la cuenca alta (fría). Las precipitaciones son casi nulas en la cuenca baja y en la alta es lluviosa de diciembre a marzo y seca de mayo a noviembre, distinguiéndose dos estaciones: la invernal y la estival (ONERN, 1975). 


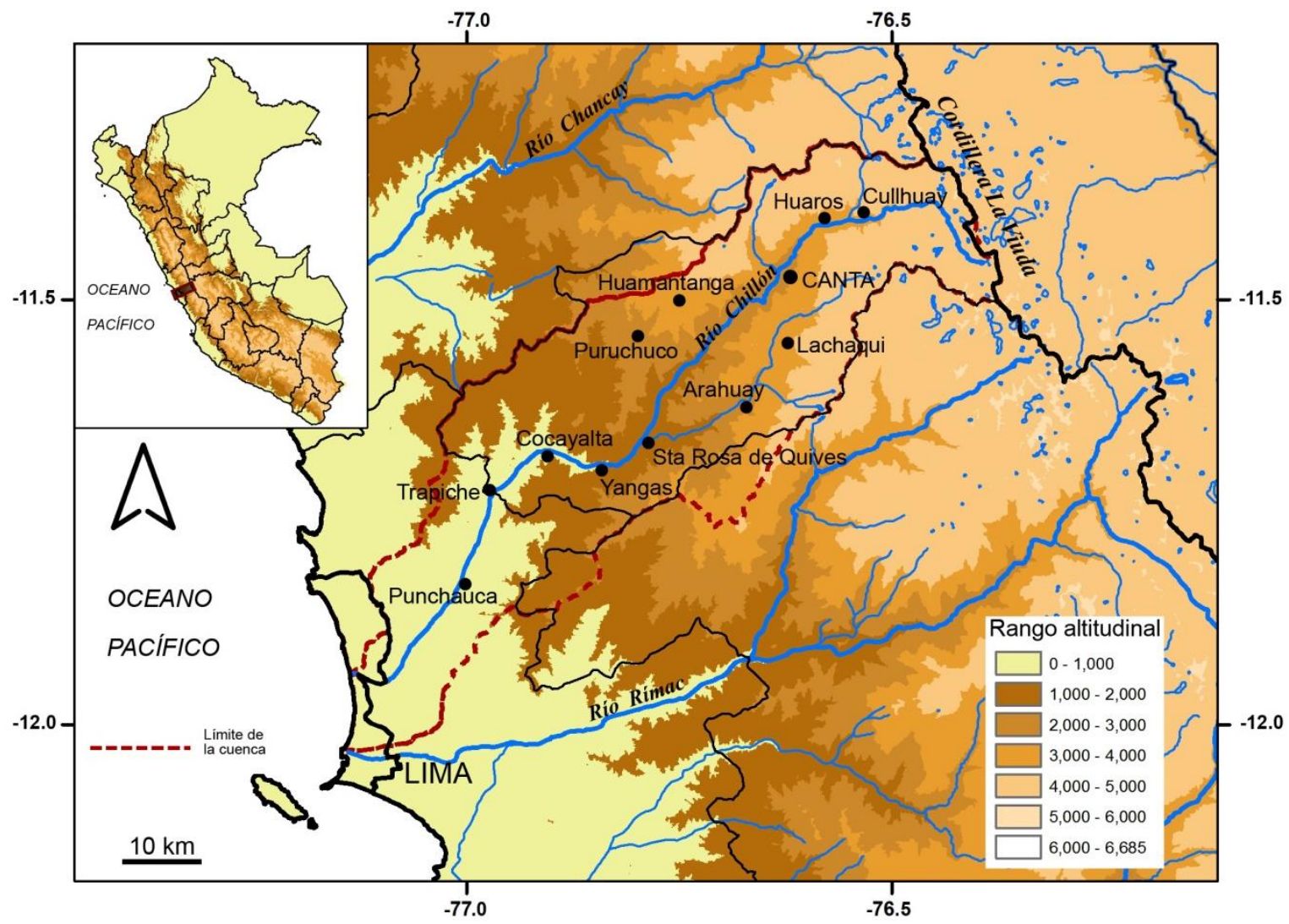

Figura 1. Mapa de ubicación de la zona de estudio.

Formaciones Vegetales.

En la cuenca en estudio se distinguen las siguientes formaciones vegetales (Weberbauer, 1945):

Piso desértico $(540-1000 \mathrm{msnm})$, incluye las localidades de Trapiche, Huanchipuquio, Zapán, Macas, Cocayalta y Yangas, de clima semidesértico, la precipitación es casi nula durante el año. La vegetación en las pendientes rocosas es escasa, están presentes algunas cactáceas dispersas, las especies típicas son, Encelia canescens, Syncretocarpus sericeus, Trixis cacalioides y Onoseris odorata.

Piso de cactáceas columnares $\left(\begin{array}{lll}1 & 000 & -2 \\ 2 & 200\end{array}\right.$ msnm), comprende las localidades de Santa Rosa de Quives, Apán y Yaso. Con escasa precipitación de enero a marzo, la vegetación se caracteriza por el predominio de cactáceas columnares y vegetación herbácea en la estación invernal. Destacando Neoraimondia arequipensis y Armatocereus matucanensis por su porte erguido. En el cerro Umarcata de Santa Rosa de Quives, entre los 1260 - 1 350 msnm, son comunes Ambrosia peruviana, Baccharis salicifolia, Bidens exigua, Onoseris odorata, Ophryosporus galioides, Pluchea chingoyo, Simsia dombeyana, Verbesina saubinetioides y en las localidades de Apán y Yaso, Heiseria pusilla, Jungia axillaris, Lomanthus tovarii, Mikania micrantha, Notobaccharis candolleana, Proustia cuneifolia.
Monte ribereño (540 - $2400 \mathrm{msnm}$ ), constituida por la vegetación a orillas del rio, es una formación vegetal siempre verde por la presencia de arbustos y algunos árboles. Durante la estación seca permanece siempre verde por la presencia de Gynerium sagittatum, Tessaria integrifolia, Cotaderia sellowiana, Enydra sessiliflora, Picrosia longifolia, Spilanthes leiocarpa, contrastando con el paisaje seco de las laderas.

Piso herbáceo de gramíneas con arbustos dispersos (2 200 - $3100 \mathrm{msnm}$ ), comprende las localidades de San Lorenzo, Viscas, San José, San Buenaventura, Carhua y Canta, caracterizado por clima templado, moderadamente húmedo, con viento frío $\mathrm{y}$ seco, con dos estaciones diferenciadas, un verano lluvioso e invierno caluroso. La vegetación está conformada por arbustos dispersos, escasos matorrales y hierbas anuales y perennes como, Baccharis arguta, Baccharis spartea, Barnadesia dombeyana, Chionopappus benthamii, Coreopsis pickeringii, Jungia pauciflora, Munnozia lyrata, Ophryosporus floribundus, Paracalia jungioides, Perymenium jelskii, Phyloglossa peruviana, Verbesina hastifolia, Wedelia helianthoides y Zinnia peruviana, entre otras. Entre los 2700 - 3100 msnm en los distritos de Arahuay, San Buenaventura y Canta, se determinaron, Achyrocline ramosissima, Aldama helianthoides, Ambrosia arborescens, Aristiguietia discolor, Baccharis alaternoides, Baccharis gnidiifolia, Baccharis latifolia, 
Dasyphyllum ferox, Jungia schuerae, Lomanthus cantensis, Lomanthus velardei, Onoseris albicans, Ophryosporus mathewsii, Ophryosporus peruvianus.

Monte rígido (3 100 - 3900 msnm), comprende los distritos de Huamantanga, San Buenaventura, Canta, Huaros, Lachaqui y Arahuay. Piso de clima frío, vientos secos, con dos estaciones bien diferenciadas, invierno caluroso con heladas nocturnas y verano lluvioso. La vegetación es más densa, conformada por arbustos y abundantes herbáceas, caracterizados por la rigidez de sus hojas y presencia de resinas. Las especies comunes son, Achyrocline alata, Ageratina sternbergiana, Baccharis buxifolia, Bidens andicola, Coreopsis fasciculata, Chaetanthera peruviana, Gynoxys visoensis, Heliopsis buphthalmoides, Hieracium leptocephalium, Hypochaeris chillensis, Hypochaeris meyeniana, Lomanthus calachaquensis, Lomanthus yauyensis, Mutisia acuminata, Ophryosporus ferreyrii, Paranephelius ovatus, Senecio breviscapus, $S$. collinus, $S$. comosus, $S$. culcitioides, $S$. condimentarius, $S$. gracilipes, $S$. richii, entre otras.

Estepa de gramíneas o puna (3 900 - 4800 msnm), de clima húmedo, viento frío y helado, la temperatura en las noches de invierno desciende bajo cero, el verano es lluvioso con truenos y relámpagos. La vegetación predominante son las gramíneas y arbustos bajos dispersos. En este piso distinguimos la vegetación de los bofedales, roquedales y pajonales. Las especies típicas son, Baccharis alpina, Cuatrecasasiellla isernii, Chersodoma antenaria, Facelis plumosa, Hypochaeris taraxacoides, Mniodes pulvinata, Misbrokea strigosissima, Novenia acaulis, Parastrephia quadrangularis, Perezia coerulescens, Perezia multiflora, Senecio calvus, S. canescens, S. casapaltensis, S. danai, S. genesianus, S. hohenackeri, $S$. nivalis, S. nutans, S. pyrenophilus, S. rhizomatus, Werneria caespitosa, W. nubigena, W. orbignyana, W. pygmaea, W. villosa, Xenophyllum dactylophyllum, X. decorum, $X$. poposum y X. staffordiae.

\section{Materiales y métodos.}

La autora principal en la cual está basada la mayor información colectó desde el año 1996 hasta el 2006; luego, esporádicamente, Hamilton Beltrán hasta inicios del 2017. La técnica de colección fue la convencional (Cerrate, 1964), para el proceso de determinación se consultó herbarios nacionales (CUZ, CPUN, HAO, HUSA, HUT, MOL, USM) y extranjeros (MO, F, US, LP), complementariamente se consultó bibliografía especializada. También se consultó la informacion en línea (web) de diferentes herbarios, tales como F, US, MO, P, en las cuales se encuentran un gran legado de las colecciones de H. Ruiz y J. Pavón, L. Nee y T. Haenke, E. F. Poeppig, A. Cruckshank, A. Mathews, expedición Wilkes, F. Pennell, realizadas desde 1778 hasta 1925. En el Herbario USM y en el MOL se revisaron las colecciones para la cuenca del río Chillón de los botánicos R. Ferreyra, O. Tovar, C. Acleto, I. Meza, M. Flores, P. Gonzáles y A. Granda.

Las especies presentadas en este trabajo han sido verificadas físicamente de tal forma que el nombre está asociado a una planta colectada en la cuenca del río Chillón u observada por los autores en este lugar. Asimismo, se ha realizado una revisión exhaustiva del protólogo de cada nombre para conocer los sinónimos.

Para cada especie se menciona el nombre válidamente publicado, distribución actualizada en el Perú por departamentos y el rango altitudinal (Tabla 1).

La clasificación está de acuerdo a Bremer (1994), a excepción de la tribu Inuleae circunscrito aquí en sentido amplio (Incluyendo Gnaphalieae, Plucheeae).

\section{Resultados y conclusiones.}

Para la cuenca del río Chillón se registran 235 (especies nativas y/o naturalizadas (no cultivadas) agrupadas en 95 géneros y 12 tribus (Tabla 1). A nivel taxonómico de Tribu: Senecioneae (62 spp.), Heliantheae (47 spp.), Inuleae (24 spp.), Eupatorieae (22 spp.), Astereae (21 spp.), Mutisieae (19 spp.), Lactuceae (13 spp.), Heleniae (10 spp.), Liabeae (5 spp.), Barnadesieae (5 spp.), Anthemidae (6 spp.) y Cardueae (1 spp.), las dos primeras tribus tienen el mayor número de especies y son valores esperados debido a su representatividad en ambos hemisferios y especialmente en los Andes sudamericanos. En la categoría de género, de los 95 registrados: Senecio $(32$ spp.), Baccharis (10 spp.), Lomanthus (9 spp.) y Werneria (9 spp.) reúnen la mayor riqueza de especies y 53 géneros están representados por una sola especie.

En relación a la distribución en el contexto de la flora del mundo, de las 235 especies en la zona de estudio, $172(73.19 \%)$ tienen amplia distribución, es decir, conocida en otros países de sudamérica y del mundo y, 63 (26.80\%) conocidas sólo en el Perú.

Las Asteráceas registradas en la Cuenca del rio Chillón (Lima) y al compararlos su ocurrencia con los otros departamentos, se evidencian cuatro grupos: el primero con especies de amplia distribución desde Tumbes hasta Tacna y Puno con 106 como Baccharis salicifolia, B. genistelloides y B. latifolia, las cuales están presentes en más de 18 departamentos; el segundo grupo, las del norte, desde Lima hasta Tumbes con 26 especies entre las que están Perymenium jelskii, Ophryosporus galioides, Jungia schuerae $y$ Ophryosporus hartwegii; el tercer grupo, cuya distribución está desde Lima hasta Puno con 77 especies como Chersodoma juanisernii, Senecio condimentarius, Xenophyllum digitatum, Stuckertiella capitata y Achyrocline ramosissima; y el cuarto desde Ancash hasta Huancavelica con 26 especies, de las cuales ocho son endémicas de Lima y dentro de éstas están Ophryosporus mathewsii y Pentacalia poeppigiana hasta ahora reportados solamente para la provincia de Canta. Lo mencionado podría ser relevante para dar inicio de como las Asteráceas están 
distribuidas en el Perú, no en función de limites departamentales, sino latidudinalmente, altitudinalmente o por cuencas hidrográficas, pero antes, debe tener prioridad el nombre válido de las especies, es decir, haber revisado la nomenclatura, sinónimos etc, para evitar nombres superfluos, del mismo modo tener extrema precaución al obtener la información en línea (web), debido a que muchos pliegos no han sido revisados por los especialistas (Goodwin et al., 2015). Britto \& Arana (2014), propusieron una hipótesis biogeográfica del Perú basado en la distribución de asteráceas, con 1669 especies cuya lista no ha sido publicada, razón por la cual no se puede realizar comparaciones.

En la distribución vertical, desde el nivel del mar hasta los $5548 \mathrm{msnm}$, hay pocas especies con amplio rango altitudinal, entre las que están: Senecio vulgaris, Gamochaeta americana y Cotula australis; crecen desde los 50 - 3900 msnm. La gran mayoría están restringidas a rangos altitudinales estrechos, pero la mayor concentración ocurre entre los 3000 a 4000 msnm, debido a las condiciones favorables del clima, a la mayor disponibilidad de agua y a la oscilación de la temperatura que favorecen al desarrollo de matorrales, bosquecillos de Polylepis y establecimiento de cultivos. La más baja concentración de especies esta entre los 0 - $1000 \mathrm{msnm}$, caracterizado por la escasez de agua, algunas especies como Encelia canescens, Syncretocarpus sericeus, Trixis cacalioides, Onoseris odorata, Jungia axillaris, Baccharis salicifolia son tipicas, y por encima de los 5000 msnm, que corresponde a la vegetación crioturbada con temperaturas extremas durante el día y la noche algunas especies, como Baccharis alpina, Oriastrum cochlearifolium, Senecio calvus, $S$. candollii, $S$. canescens $S$. genisianus, $S$. nivalis, Werneria carnulosa, Xenophyllum dactylophyllum y X. poposum, crecen en las alturas más elevadas. Al comparar altitudinalmente en fajas de 500 metros con Gonzáles (2016), son parecidos tanto en especies como en cantidades y en el rango de los 3000 a 4000 m están por encima de las 120 especies.

Son nuevos registros para la cuenca del Chillón; Achyrocline ramosissima, Ageratina lobulifera, Cotula coronipifolia, Cotula mexicana, Enhydra sessilifolia, Novenia acaulis, Verbesina andina, Pseudognaphalium vira-vira, Senecio saxipunae y Werneria carnulosa, que no han sido previamente citados en investigaciones anteriores.

En la cuenca del rio Chillón se han descrito un apreciable número de Asteráceas como producto de la expedición del Capitán Wilkes (Wilkes, 1845), Senecio danai, Senecio pickeringii, S. richii, Coreopsis pickeringii, Senecio gracilipes, Ophryosporus mathewsii (=Piqueria mathewsii), Munnozia lyrata (=Liabum lyratum), Heiseria pusilla (=Tithonia pusilla), Chaetanthera peruviana, Lomanthus subcandidus (=Senecio subcandidus), entre las últimas en ser descrita se encuentran los basónimos de Lomanthus velardei (1950) y Lomanthus cantensis (1954), asi como Pentacalia poeppigiana (Granda, 2009).

La cuenca del río Chillón forma parte de las Provincias de Lima y Canta, la mayor superficie corresponde a esta última en la cual se han realizado las mayores colectas de Asteráceas, entre los 500 y 4800 msnm. Para la Provincia de Canta, Meza (1966) registró 92 especies colectada solo en los alrededores del pueblo de Canta y Obrajillo, Gonzáles (2016) reporta 178 especies, aunque figuran más debido a sinónimos o especies que no hay para Perú, en el presente trabajo se registran 235 especies; de éstas, 197 han sido colectadas por G. Vilcapoma (1996-2006), que se conservan en herbarios nacionales. Al comparar los tres estudios es indiscutible que el presente trabajo, corresponde a una mayor área y definido solo a la cuenca, además de una intensa colecta y trabajo de curatoria en los herbarios por muchos años. Sin embargo, aún debe haber más especies, especialmente en las partes más elevadas sobre suelos crioturbados.

\section{Agradecimientos.}

Los autores agradecen a los Directores de los Herbarios MOL y USM por permitirnos examinar las colecciones que conservan. De la misma manera, agradecemos al Fondo Especial de Desarrollo Universitario (FEDU) - UNALM por la ayuda económica para recolectar la flora de la cuenca en estudio. También expresamos nuestro reconocimiento a la Magister Mercedes Flores, a los biólogos J.J. Alegría, A. Granda, D. Huamán y Rocío Moreyra por su valiosa colaboración en el trabajo de campo. Asimismo, hacemos extensivo nuestro agradecimiento a Reynaldo Álvarez Grillo por tipear la base de datos del herbario MOL.

\section{Literatura citada.}

Ball J. 1885. Contributions to the flora of the Peruvian Andes, with remarks on the history and origin of the Andean flora. The Journal of the Linnean Society Botany. 22: $1-64$

Beltrán H. 2016. Las Asteráceas (Compositae) del distrito de Laraos (Yauyos, Lima, Perú). Revista Peruana de Biología 23: 195-220.

Beltrán H., Granda A., León B., Sagástegui A., Sánchez I. \& Zapata M. 2006. Asteraceae endémicas del Perú. Revista Peruana de Biología. 13: 64-164.

Brako L. \& Zarucchi J. 1993. Catálogo de las angiospermas y gimnospermas del Perú. Monographs in Systematic Botany from Missouri Botanical Garden. 45: 1-1286.

Bremer K. 1994. Asteraceae: Cladistic and Classification. Timber Press. Portland - Oregon.

Britto B. \& Arana C. 2014. Corotipos preliminares de Perú basados en la distribución de la familia Asteraceae. Darwiniana 2(1): 39-56. DOI: 10.14522/darwiniana.2014.21.553. 
Cano A., Delgado A., Mendoza W., Trinidad H., Gonzáles P., La Torre M.I., Chanco M., Aponte H., Roque J., Valencia N. \& Navarro E. 2011. Flora y vegetación de suelos crioturbados y hábitats asociados en los alrededores del abra Apacheta, Ayacucho - Huancavelica (Perú). Revista Peruana de Biología. 18: 169-178.

Cano A., Mendoza W., Castillo S., Morales M., La Torre M.I., Aponte H., Delgado A., Valencia N. \& Vega N. 2010. Flora y vegetación de suelos crioturbados y hábitat asociados en la Cordillera Blanca, Ancash, Perú. Revista Peruana de Biología. 17: 95-103.

Castillo G. 2006. Diversidad y distribución de Asteráceas en el Parque Nacional Yanachaga-Chemillen. Tesis Biólogo en Universidad Nacional San Agustín de Arequipa.

Cerrate E. 1964. Manera de preparar plantas para un herbario. Museo Historia Natural. Serie de Divulgación 1: 242-246.

De La Cruz A. 1999. Asteráceas de las pampas y lomas: Manzano, Pucara y Lúcumo. Prov. Huarochirí - Lima. Tesis Grado de Magister en Facultad de Ciencias Biológicas. Universidad Nacional Mayor de San Marcos.

Gonzáles P. 2016. Riqueza y distribución de Asteráceas en el departamento de Lima (Perú). Arnaldoa 23: 111-134.

Goodwin Z.A., Harris D. J., Filer D, Wood J.R.I. \& Scotland R. W. 2015. Widspread Mistaken identity in tropical plants collections - Current biology CB 25: r1066-7.

Granda A.P. 2009. Pentacalia poeppigiana (Asteraceae, Senecioneae), una nueva especie del Perú. Darwiniana 47(2): 321-326.

Meza I. 1966. Contribución al conocimiento de las compuestas de Canta. Tesis de bachiller en Facultad de Ciencias Biológicas. Universidad Nacional Mayor de San Marcos.
ONERN (Oficina Nacional de Evaluación de Recursos Naturales). 1975. Informe técnico del proyecto de irrigación Marcapomacocha. Perú.

.Rivera P. 1981. Análisis sobre las posibilidades de recreación en áreas naturales en la Cuenca del Río Chillón (Dpto. Lima - Perú). Universidad Nacional Agraria de La Molina, Perú.

Smith D.N. 1989. Flora and vegetation of the Huascarán National Park, Ancash, Peru: with preliminary taxonomic studies for a manual of the flora. Retrospective Theses and Dissertations. Iowa State University Ames, Iowa.

Spichiger R., Savolainen V., Figeat M. \& Jeanmonod D. 2002. Botanique Systématique des plantes à fleurs. Presses Polytechniques et Universitares Romandes. Francia.

Ulloa C., Acevedo-Rodríguez P., Beck S., Belgrano M.J., Bernal R., Berry P.E., et al. 2017. An integrated assessment of the vascular plant species of the Americas. Science 358: 1614-1617.

Weberbauer A. 1945. El mundo vegetal de los Andes peruanos. Ministerio de Agricultura. Lima. 776 pp.

Wilkes Ch. 1845. Narrative of the United States Exploring Expedition. London. Whitaker and Co. Av. Maria Lane.

Yarupaitán G. \& Albán J. 2003. Flora silvestre de los Andes centrales del Perú: un estudio en la zona de Quilcas, Junín. Revista peruana de Biología. 10(2): 155-162.

Tabla 1. Lista de Especies colectadas en la cuenca del rio Chillón. Las abreviaturas departamentales siguen a Brako \& Zarucchi (1993).

\begin{tabular}{|c|c|c|c|}
\hline TRIBU: ESPECIE & $\begin{array}{c}\text { Rango } \\
\text { Altitudinal } \\
\end{array}$ & $\begin{array}{c}\begin{array}{c}\text { Distribución Perú } \\
\text { (departamentos) }\end{array} \\
\end{array}$ & Colector \\
\hline \multicolumn{4}{|l|}{ Anthemidae } \\
\hline Cotula australis (Sieber ex Spreng.) Hook. f. & $2000-3800$ & $\begin{array}{l}\mathrm{am} / \mathrm{an} / \mathrm{ar} / \mathrm{ca} / \mathrm{cu} / \mathrm{hu} / \mathrm{ju} / \mathrm{la} \\
/ \mathrm{li} / \mathrm{ll} / \mathrm{mo} / \mathrm{pi} / \mathrm{pu} / \mathrm{hu} / \mathrm{ta} /\end{array}$ & VG: 2230 \\
\hline Cotula coronipifolia L. $^{1}$ & $3300-3400$ & $\mathrm{ar} / \mathrm{cu} / \mathrm{li} / \mathrm{ll} / \mathrm{pu}$ & VG: 7946 \\
\hline Cotula mexicana (DC.) Cabrera ${ }^{1}$ & $2600-4800$ & $\begin{array}{l}\mathrm{am} / \mathrm{ca} / \mathrm{cu} / \mathrm{ju} / \mathrm{li} / \mathrm{mo} / \mathrm{pu} / \mathrm{ta} \\
/ \mathrm{sm}\end{array}$ & VG: 7477 \\
\hline Leucanthemum vulgare Lamarck & $2900-3000$ & $\mathrm{cu} / \mathrm{li}$ & Visto \\
\hline Tanacetum parthenium (L.) Sch. Bip. & $2500-3500$ & $\begin{array}{l}\mathrm{am} / \mathrm{an} / \mathrm{ar} / \mathrm{ay} / \mathrm{ca} / \mathrm{cu} / \mathrm{hu} / \mathrm{li} \\
/ 11 / \mathrm{mo} / \mathrm{pu} / \mathrm{sm}\end{array}$ & Visto \\
\hline $\begin{array}{l}\text { Soliva stolonifera (Brot.) Sweet } \\
\text { Asteraeae }\end{array}$ & $3800-4500$ & li & VG: 5697 \\
\hline Baccharis alaternoides Kunth & $3000-3500$ & an/ay/ca/cu/hv/li/ll & VG: 4753 \\
\hline Baccharis alpina Kunth & $3800-4800$ & $\begin{array}{l}\text { an/ar/ay/ca/cu/hv/ju/li/ } \\
\mathrm{mo} / \mathrm{pa} / \mathrm{pu} / \mathrm{ta}\end{array}$ & VG: 2505 \\
\hline Baccharis arguta Gillies ex Hook. \& Arn. & $2000-3700$ & an/ap/ay/cu/hu/li/pu & VG: 2224 \\
\hline Baccharis buxifolia (Lam.) Pers. & $3600-4500$ & $\begin{array}{l}\mathrm{am} / \mathrm{an} / \mathrm{ap} / \mathrm{ar} / \mathrm{ay} / \mathrm{ca} / \mathrm{cu} / \mathrm{h} \\
\mathrm{u} / \mathrm{ju} / \mathrm{la} / \mathrm{li} / \mathrm{ll} / \mathrm{pu} / \mathrm{ta}\end{array}$ & VG: 1239 \\
\hline Baccharis caespitosa (Ruiz \& Pav.) Pers. & $3900-4800$ & $\begin{array}{l}\text { an/ar/ay/ca/cu/hu/hv/ju } \\
/ \mathrm{la} / \mathrm{li} / \mathrm{ll} / \mathrm{mo} / \mathrm{pu} / \mathrm{sm} / \mathrm{ta}\end{array}$ & VG: 4499 \\
\hline Baccharis genistelloides (Lam.) Pers. & $2000-5000$ & $\begin{array}{l}\text { an/ay/ap/ar/ca/cu/hu/hv } \\
\text { /ju/la/ll/li/mo/pa/pi/pu/ } \\
\text { sm/ta }\end{array}$ & VG: 1497 \\
\hline Baccharis gnidiifolia Kunth & $2100-3500$ & $\begin{array}{l}\text { am/an/ar/ay/ca/cu/la/lii/ } \\
\text { 11/mo/pi/ta }\end{array}$ & VG: 4845 \\
\hline
\end{tabular}


Tabla 1. Lista de Especies colectadas en la cuenca del rio Chillón. Las abreviaturas departamentales siguen a Brako \& Zarucchi (1993).

\begin{tabular}{|c|c|c|c|c|}
\hline TRIBU: ESPECIE & & $\begin{array}{c}\text { Rango } \\
\text { Altitudinal }\end{array}$ & $\begin{array}{c}\begin{array}{c}\text { Distribución Perú } \\
\text { (departamentos) }\end{array} \\
\end{array}$ & Colector \\
\hline Baccharis latifolia (Ruiz \& Pav.) Pers. & & $100-3900$ & $\begin{array}{l}\text { am/an/ap/ar/ay/ca/cu/h } \\
\text { u/hv/ic/ju/li/la/ll/pa/pi/ } \\
\mathrm{pu} / \mathrm{sm}\end{array}$ & VG: 7129 \\
\hline Baccharis salicifolia (Ruiz \& Pav.) Pers. & & $100-2900$ & $\begin{array}{l}\text { am/an/ar/ap/ay/ca/cu/h } \\
\mathrm{u} / \mathrm{hv} / \mathrm{ic} / \mathrm{ju} / \mathrm{li} / \mathrm{la} / \mathrm{ll} / \mathrm{lo} / \mathrm{md} / \\
\mathrm{pa} / \mathrm{pi} / \mathrm{pu} / \mathrm{sm} / \mathrm{uc}\end{array}$ & VG: 4858 \\
\hline Baccharis spartea Benth. & Figura 2 & $3000-3500$ & $\begin{array}{l}\text { an/ar/ay/ca/cu/la/li/ll/m } \\
\text { o }\end{array}$ & VG: 4190 \\
\hline Conyza artemisiifolia Meyen \& Walp. & & $2500-4600$ & $\begin{array}{l}\text { an/ar/ca/cu/hv/ju/la/la/l } \\
\text { 1/li/lo/mo/pu/ta/sm/uc }\end{array}$ & VG: 6015 \\
\hline Conyza bonariensis (L.) Cronquist & & $390-3900$ & $\begin{array}{l}\mathrm{am} / \mathrm{an} / \mathrm{ar} / \mathrm{ca} / \mathrm{cu} / \mathrm{hu} / \mathrm{ic} / \mathrm{ju} \\
/ \mathrm{li} / \mathrm{lo} / \mathrm{mo} / \mathrm{pa} / \mathrm{pu} / \mathrm{sm} / \mathrm{tu} / \mathrm{u} \\
\mathrm{c}\end{array}$ & VG: 1753 \\
\hline Conyza canadensis (L.) Cronquist & & $430-2600$ & $\mathrm{am} / \mathrm{cu} / \mathrm{hu} / \mathrm{ju} / \mathrm{li} / \mathrm{md}$ & GP: 643 \\
\hline Conyza coronopifolia Kunth & & $3300-4200$ & $\begin{array}{l}\text { an/ay/cu/hv/hu/ju/la/li/ } \\
\mathrm{mo} / \mathrm{pa} / \mathrm{pu}\end{array}$ & VG: 8028 \\
\hline Conyza sumatrensis (Retz.) E. Walker & & $2400-3500$ & $\begin{array}{l}\text { am/an/ar/cu/ca/hu/hv/j } \\
\text { u/li/mo }\end{array}$ & VG: 4881 \\
\hline Erigeron ecuadoriensis Hieron. & & $2700-3700$ & $\mathrm{am} / \mathrm{an} / \mathrm{hu} / \mathrm{ju} / \mathrm{li} / \mathrm{pi} / \mathrm{sm}$ & VG: 5393 \\
\hline Erigeron leptorhizon DC.* & & $5-1000$ & an/hv/ic/la/li/ll & VG: 5584 \\
\hline Erigeron rosulatus Wedd. & & $3500-4900$ & $\begin{array}{l}\text { an/ay/ar/cu/hv/li/mo/pu } \\
/ \mathrm{sm} / \mathrm{ta}\end{array}$ & VG: 4479 \\
\hline Novenia acaulis (Wedd. ex Benth.) Freire \& Hellwig ${ }^{1}$ & Figura 3 & $3900-4500$ & $\begin{array}{l}\text { an/ay/ca/cu/hu/ju/li/ll/p } \\
\text { u/pa }\end{array}$ & VG: 4148 \\
\hline Oritrophium limnophilum (Sch.Bip.) Cuatrec. & & $3900-4900$ & $\begin{array}{l}\text { an/ar/ay/ca/hu/ju/li/ll/p } \\
\text { a/pu/sm }\end{array}$ & GP: 773 \\
\hline Parastrephia quadrangularis (Meyen) Cabrera & Figura 4 & $3200-4900$ & $\begin{array}{l}\text { ar/ay/cu/hv/ju/li/mo/pu } \\
/ \mathrm{ta}\end{array}$ & VG: 5921 \\
\hline \multicolumn{5}{|l|}{ Barnadesieae } \\
\hline Barnadesia dombeyana Less.* & & $2700-4000$ & an/ap/ca/hu/ll/li/pa & RF: 18415 \\
\hline Barnadesia lehmannii Hieron. & & $3000-3700$ & an/li/ & VG: 8056 \\
\hline Barnadesia reticulata D. Don* & & $2700-3000$ & $\mathrm{ic} / \mathrm{li} / \mathrm{ju}$ & RF: 6914 \\
\hline Chuquiraga spinosa Less. & & $3900-4800$ & $\begin{array}{l}\text { an/ap/ay/cu/hu/hv/ju/li/ } \\
\mathrm{pa} / \mathrm{pu}\end{array}$ & VG: 2580 \\
\hline $\begin{array}{l}\text { Dasyphyllum ferox (Wedd.) Cabrera } \\
\text { Cardueae }\end{array}$ & Figura 5 & $3000-3900$ & an/cu/hv/ju/li/la/ll & VG: 2592 \\
\hline \multicolumn{4}{|l|}{ Eupatorieae } & VG: 4835 \\
\hline Ageratina glechonophylla (Less.) R.M.King \& H. Rob. & & $2100-4800$ & $\begin{array}{l}\text { an/ay/ca/cu/hu/ju/li/ll/ } \\
\mathrm{mo} / \mathrm{pu} /\end{array}$ & VG: 649 \\
\hline Ageratina lobulifera (B. Rob.) R.M.King \& H. Rob.*1 & & $3000-4000$ & an/li/mo & VG: 2527 \\
\hline Ageratina sternbergiana (DC.) R.M. King \& H. Rob. & & $1000-4100$ & $\begin{array}{l}\text { an/ap/ay/ar/ca/cu/hu/hv } \\
/ \mathrm{ju} / \mathrm{li} / \mathrm{ll} / \mathrm{mo} / \mathrm{pa} / \mathrm{pu}\end{array}$ & VG: 7799 \\
\hline Ageratum conyzoides $\mathrm{L}$. & & $5-1000$ & $\begin{array}{l}\mathrm{am} / \mathrm{an} / \mathrm{ay} / \mathrm{ca} / \mathrm{cu} / \mathrm{hu} / \mathrm{ic} / \mathrm{ju} \\
/ \mathrm{li} / \mathrm{ll} / \mathrm{lo} / \mathrm{md} / \mathrm{pa} / \mathrm{pi} / \mathrm{sm} / \mathrm{uc}\end{array}$ & WE \\
\hline Aristeguietia discolor (DC.) R.M. King \& H. Rob.* & & $3000-3900$ & $\begin{array}{l}\text { an/ap/ay/ca/cu//hv/hu/j } \\
\mathrm{u} / \mathrm{la} / \mathrm{li} / \mathrm{ll} / \mathrm{pa} / \mathrm{sm}\end{array}$ & VG: 1770 \\
\hline Cronquistianthus glomeratus (DC.) R.M.King \& H. Rob.* & & $2700-3500$ & an/ay/ca/hu/hv/li & VG: 5168 \\
\hline Helogyne virgata (Rusby) B.L. Rob. & & $1000-2400$ & an/ic/li & GP: 1725 \\
\hline Mikania micrantha Kunth & & $500-3000$ & $\begin{array}{l}\mathrm{am} / \mathrm{ca} / \mathrm{cu} / \mathrm{hu} / \mathrm{la} / \mathrm{li} / \mathrm{lo} / \mathrm{md} \\
/ \mathrm{pa} / \mathrm{pi} / \mathrm{sm} / \mathrm{uc}\end{array}$ & VG: 1046 \\
\hline Nothobaccharis candolleana (Steud.) R.M.King \& H. Rob.** & & $1900-2700$ & li & VG: 478 \\
\hline Ophryosporus apricus B.L. Rob.* & & $2000-3000$ & $\mathrm{am} / \mathrm{ca} / \mathrm{hu} / \mathrm{li}$ & IM: 212 \\
\hline Ophryosporus ferreyrii H. Rob.** & & $3200-3900$ & li & VG: 7027 \\
\hline Ophryosporus floribundus (DC.) R.M. King \& H. Rob. & & $800-3000$ & ic/li & VG: 2388 \\
\hline Ophryosporus galioides (DC.) R.M. King \& H. Rob.* & & $150-2000$ & an/ca/la/li/pi/sm & VG: 2391 \\
\hline Ophryosporus hartwegii (B. Rob.) R.M. King \& H. Rob.* & & $2900-2900$ & $\mathrm{ca} / \mathrm{ll} / \mathrm{li}$ & GP: 1716 \\
\hline Ophryosporus mathewsii (B. Rob.) R.M. King \& H. Rob.*** & & $2200-3000$ & li & VG: 8029 \\
\hline Ophryosporus peruvianus (J. Gmel.) R.M. King \& H. Rob. & & $700-3700$ & $\begin{array}{l}\text { an/ap/ar/ay/ca/cu/hv/ic/ } \\
\text { la/li/ll/mo/pi/ta }\end{array}$ & VG: 1657 \\
\hline
\end{tabular}


Tabla 1. Lista de Especies colectadas en la cuenca del rio Chillón. Las abreviaturas departamentales siguen a Brako \& Zarucchi (1993).

\begin{tabular}{|c|c|c|c|}
\hline TRIBU: ESPECIE & $\begin{array}{c}\text { Rango } \\
\text { Altitudinal }\end{array}$ & $\begin{array}{c}\begin{array}{c}\text { Distribución Perú } \\
\text { (departamentos) }\end{array} \\
\end{array}$ & Colector \\
\hline Ophryosporus pubescens (Smith) R.M. King \& H. Rob.* & $500-2500$ & ar/ic/li/ & GP: 947 \\
\hline Phalacraea latifolia DC.** & $2100-3400$ & li & GA: 2238 \\
\hline Stevia macbridei B.L. Rob. & $2000-3800$ & $\begin{array}{l}\text { an/cu/ca/hu/hv/ju/la/li/l } \\
\text { l/mo/pu }\end{array}$ & VG: 2633 \\
\hline Stevia melissiaefolia (Lam.) Sch.Bip.* & $200-900$ & $\mathrm{ar} / \mathrm{ca} / \mathrm{cu} / \mathrm{li}$ & VG: 5938 \\
\hline Stevia petiolata (Cass.) Sch.Bip.* & $2000-3800$ & an/cu/li/ & VG: 1595 \\
\hline Stevia puberula Hook.* & $2000-3500$ & $\begin{array}{l}\text { am/an/ay/cu/hv/li/ll/la/ } \\
\text { pi }\end{array}$ & VG: 2335 \\
\hline \multicolumn{4}{|l|}{ Helenieae } \\
\hline Flaveria bidentis (L.) Kuntze & $50-1000$ & $\begin{array}{l}\text { ar/ay/ca/hv/ic/ju/li/ll/m } \\
\text { o/ta }\end{array}$ & VG: 2413 \\
\hline Microseris pygmaea D. Don & $3800-4500$ & an/li/ta & VG: 2700 \\
\hline Pectis sessiliflora (Less.) Sch.Bip. ex Rusby & $2000-3000$ & an/ay/cu/hu/hv/li/pi/ & VG: 7924 \\
\hline Porophyllum ruderale (Jacq.) Cass. & $5-3200$ & $\begin{array}{l}\mathrm{am} / \mathrm{an} / \mathrm{ca} / \mathrm{cu} / \mathrm{hu} / \mathrm{hv} / \mathrm{ju} / \mathrm{la} \\
/ \mathrm{li} / \mathrm{ll} / \mathrm{lo} / \mathrm{md} / \mathrm{pi} / \mathrm{sm} / \mathrm{tu} / \mathrm{uc}\end{array}$ & VG: 4170 \\
\hline Schkuhria pinnata (Lam.) Kuntze & $1000-3000$ & $\begin{array}{l}\text { an/ay/ca/cu/hu/li/li/pi/ } \\
\text { mo }\end{array}$ & VG: 2268 \\
\hline Tagetes elliptica Smith & $50-3800$ & $\mathrm{am} / \mathrm{an} / \mathrm{hu} / \mathrm{ju} / \mathrm{li} / \mathrm{mo} / \mathrm{pa}$ & VG: 1763 \\
\hline Tagetes filifolia Lag. & $2000-3800$ & $\begin{array}{l}\text { am/an/ar/ay/ca/cu/hu/ju } \\
/ \text { li /l1/pa/pi/pu }\end{array}$ & VG: 5337 \\
\hline Tagetes gracilis DC. & $2000-3800$ & an/ar/ay/cu/ju/li/mo/pu & VG: 2294 \\
\hline Tagetes minuta $\mathrm{L}$. & $900-3400$ & $\mathrm{ca} / \mathrm{ju} / \mathrm{li} / \mathrm{pu}$ & VG: 4843 \\
\hline Tagetes multiflora Kunth & $1000-3900$ & $\begin{array}{l}\text { an/ar/ay/ca/cu/hu/ju/li/ } \\
\mathrm{mo} / \mathrm{pu} / \mathrm{ta}\end{array}$ & VG: 4944 \\
\hline \multicolumn{4}{|l|}{ Heliantheae } \\
\hline Acanthospermum hispidum DC. & $500-2000$ & an/cu/ic/ju/li & Visto \\
\hline Acmella alba (L'Her.) R.K. Jansen & $50-3300$ & $\begin{array}{l}\mathrm{am} / \mathrm{an} / \mathrm{ca} / \mathrm{ju} / \mathrm{la} / \mathrm{li} / \mathrm{ll} / \mathrm{lo} / \mathrm{pi} \\
/ \mathrm{tu}\end{array}$ & VG: 4179 \\
\hline Acmella ciliata (Kunth) Cass. & $100-2600$ & $\begin{array}{l}\text { am/an/cu/hu/ic/ju/la/li/l } \\
\text { 1/lo/md/pa/pu/sm/uc }\end{array}$ & GP: 1574 \\
\hline Acmella oppositifolia (Lam.) R.K. Jansen & $2000-3000$ & an/li & GP: 687 \\
\hline Aldama helianthoides (Rich.) E.E.Schill. \& Panero & $2100-3900$ & $\begin{array}{l}\text { an/ay/ca/cu/hu/hv/ju/li/ } \\
\mathrm{mo} / \mathrm{pa} / \mathrm{pu} / \mathrm{ta}\end{array}$ & VG: 5991 \\
\hline Aldama lanceolata (Britton) E.E.Schill. \& Panero & $2000-3700$ & $\begin{array}{l}\text { an/ar/ay/cu/li/l1/ju/mo/ } \\
\mathrm{pu}\end{array}$ & VG: 1710 \\
\hline Aldama peruviana (A.Gray) E.E.Schill. \& Panero* & $2000-3700$ & an/ar/li/mo/ta/ & WE \\
\hline Aldama truxillensis (Kunth) S.F. Blake* & $2900-3500$ & an/ap/ca/ju/la/ll/li/pi & SG: 937 \\
\hline Ambrosia arborescens Mill. & $1000-4000$ & $\begin{array}{l}\text { am/an/cu/hu/ju/li/mo/p } \\
\text { a/pi/pu/ta }\end{array}$ & VG: 5178 \\
\hline Ambrosia peruviana All. & $1500-3000$ & $\begin{array}{l}\text { am/an/ar/ca/hu/hv/ic/la } \\
\text { /l1/li/lo/mo/pi }\end{array}$ & VG: 5598 \\
\hline Aphanactis villosa S.F. Blake & $3200-4000$ & $\mathrm{an} / \mathrm{cu} / \mathrm{hu} / \mathrm{sm} / \mathrm{ju} / \mathrm{li} / \mathrm{ll}$ & VG: 5811 \\
\hline Bidens abadiae DC. & $100-1500$ & an/li & GP: 3693 \\
\hline Bidens andicola Kunth & $3000-4500$ & $\begin{array}{l}\text { am/an/ar/ay/ca/cu/hu/h } \\
\text { v/ju/li/ll/mo/pa/pu/ta }\end{array}$ & VG: 5305 \\
\hline Bidens exigua Sherff & $2000-2500$ & $\mathrm{am} / \mathrm{ca} / \mathrm{cu} / \mathrm{la} / \mathrm{li} / \mathrm{sm}$ & VG: 5255 \\
\hline Bidens pilosa $\mathrm{L}$. & $50-3900$ & $\begin{array}{l}\text { am/an/ar/ay/ca/cu/hu/h } \\
\text { v/ic/ju/li/lo/md/pa/pi/p } \\
\text { u/sm/uc }\end{array}$ & VG: 7078 \\
\hline Bidens triplinervia Kunth & $3000-4000$ & $\begin{array}{l}\mathrm{am} / \mathrm{an} / \mathrm{ca} / \mathrm{cu} / \mathrm{hv} / \mathrm{ju} / \mathrm{li} / \mathrm{ll} / \\
\mathrm{pa} / \mathrm{pu} / \mathrm{ta}\end{array}$ & VG: 8072 \\
\hline Coreopsis fasciculata Wedd. & $2000-4000$ & $\begin{array}{l}\text { an/ap/ar/ay/hv/li/ll/mo/ } \\
\text { ta }\end{array}$ & VG: 1768 \\
\hline Coreopsis pickeringii A. Gray & $3000-3500$ & an/ca/li & VG: 2203 \\
\hline Eclipta postrata L. & $100-1000$ & $\begin{array}{l}\text { an/ar/ca/hu/ic/li/ll/lo/m } \\
\text { d/pa/sm/tu/uc }\end{array}$ & VG: 4957 \\
\hline Encelia canescens Lam. & $2000-2900$ & $\begin{array}{l}\text { an/ar/ic/la/li//1/mo/pi/ta } \\
/ \mathrm{tu}\end{array}$ & VG: 7558 \\
\hline Enydra sessilifolia (Ruiz \& Pav.) Cabrera ${ }^{1}$ & $5-100$ & $\mathrm{ic} / \mathrm{la} / \mathrm{li} / \mathrm{ll}$ & VG: 4959 \\
\hline
\end{tabular}


Tabla 1. Lista de Especies colectadas en la cuenca del rio Chillón. Las abreviaturas departamentales siguen a Brako \& Zarucchi (1993).

\begin{tabular}{|c|c|c|c|c|}
\hline TRIBU: ESPECIE & & $\begin{array}{c}\text { Rango } \\
\text { Altitudinal }\end{array}$ & $\begin{array}{c}\begin{array}{c}\text { Distribución Perú } \\
\text { (departamentos) }\end{array} \\
\end{array}$ & Colector \\
\hline Galinsoga mandonii Sch.Bip. & & $2000-4300$ & $\begin{array}{l}\text { an/ar/ay/ca/cu/hu/hv/ju } \\
\text { /la/li/mo/pu/ta }\end{array}$ & VG: 6007 \\
\hline Galinsoga parviflora Cav. & & $5-1000$ & $\begin{array}{l}\text { an/ar/ca/cu/hu/hv/ic/ju/ } \\
\mathrm{la} / \mathrm{li} / \mathrm{ll} / \mathrm{mo} / \mathrm{pi}\end{array}$ & VG: 7626 \\
\hline Galinsoga quadriradiata Ruiz \& Pav. & & $50-3800$ & $\begin{array}{l}\text { an/ca/cu/hu/ic/ju/la/li/s } \\
\mathrm{m}\end{array}$ & VG: 5150 \\
\hline Heiseria pusilla (A.Gray) E.E.Schill. \& Panero* & & $400-2800$ & an/ca/hv/la/li/ & VG: 2381 \\
\hline Heiseria simsioides (S.F.Blake) E.E.Schill. \& Panero* & & $800-3600$ & an/l1/li & GP: 1789 \\
\hline Heliopsis buphthalmoides (Jacq.) Dunal & Figura 6 & $3100-3800$ & $\begin{array}{l}\text { am/an/ap/ay/ca/cu/ju/h } \\
\mathrm{u} / \mathrm{la} / \mathrm{li} / \mathrm{ll} / \mathrm{pa}\end{array}$ & VG: 5143 \\
\hline Heterosperma diversifolium Kunth & & $800-3400$ & an/ar/cu/li/ll/pi & VG: 3256 \\
\hline Heterosperma ovatifolium Cav. & Figura 7 & $1000-2500$ & an/ar/ca/ju/li & VG: 1049 \\
\hline Perymenium jelskii (Hieron.) S.F. Blake & & $2000-2500$ & $\mathrm{am} / \mathrm{ca} / \mathrm{hu} / \mathrm{hv} / \mathrm{la} / \mathrm{li} / \mathrm{pi}$ & VG: 4670 \\
\hline Siegesbeckia agrestis Poepp. & & $500-3500$ & $\mathrm{am} / \mathrm{li}$ & GP 923 \\
\hline Siegesbeckia flosculosa L'Her. & & $1800-3000$ & $\mathrm{an} / \mathrm{ar} / \mathrm{ca} / \mathrm{cu} / \mathrm{hu} / \mathrm{li} / \mathrm{sm}$ & VG: 4859 \\
\hline Simsia dombeyana DC. & & $100-2300$ & la/li/ll/pi/tu & VG: 7575 \\
\hline Smallanthus fruticosus (Benth.) H. Rob. & & $1900-3700$ & an/ca/hu/li/l1/pi & VG: 7776 \\
\hline Smallanthus glabratus (DC.) H. Rob. & & $1800-3600$ & $\begin{array}{l}\mathrm{an} / \mathrm{ca} / \mathrm{hv} / \mathrm{hu} / \mathrm{ju} / \mathrm{ll} / \mathrm{li} / \mathrm{la} / \mathrm{pi} \\
/ \mathrm{pu}\end{array}$ & VG: 4227 \\
\hline Smallanthus microcephalus (Hieron.) H. Rob. & & $2200-3500$ & an/ca/li/hv & VG: 8061 \\
\hline Smallanthus parviceps (S.F. Blake) H. Rob. & & $1800-3600$ & $\begin{array}{l}\text { an/ap/ay/ca/cu/hv/li/pi/ } \\
\mathrm{pu}\end{array}$ & IM: 144 \\
\hline Spilanthes leiocarpa DC. & & 5- 1400 & an/ar/ic/li/ll/pi/tu & VG: 5646 \\
\hline Syncretocarpus sericeus (DC.) S.F. Blake* & & $1100-2500$ & an/ar/ca/ic/ju/li & VG: 4635 \\
\hline Verbesina andina Sagást.*1 & & $2900-3600$ & $\mathrm{hu} / \mathrm{li}$ & VG: 4165 \\
\hline Verbesina hastifolia S.F. Blake* & & $900-2500$ & an/li/l1/ & VG: 5362 \\
\hline Verbesina saubinetioides S.F. Blake* & & $600-3000$ & an/ic/li/la/ll & VG: 2245 \\
\hline Villanova oppositifolia (Lag.) S.F. Blake & Figura 8 & $50-3000$ & an/ar/ic/ju/li/mo/ta & Visto \\
\hline Villanova titicacensis (Meyen \& Walp.) Walp. & & $2300-3900$ & $\begin{array}{l}\text { an/ap/ay/ca/cu/ju/li/ll/ } \\
\mathrm{mo} / \mathrm{pu} / \mathrm{ta}\end{array}$ & VG: 5427 \\
\hline Wedelia helianthoides Kunth & & $2500-3500$ & an/ca/li/ & VG: 5263 \\
\hline Xanthium spinosum $\mathrm{L}$. & & $1800-3800$ & $\begin{array}{l}\text { am/an/ay/hu/hv/ju/li/ll/ } \\
\text { mo }\end{array}$ & VG: 5958 \\
\hline Zinnia peruviana (L.) L. & & $50-3600$ & $\begin{array}{l}\text { an/ar/ay/ca/cu/hu/hv/ju } \\
/ \mathrm{la} / \mathrm{li} / \mathrm{ll} / \mathrm{pi} / \mathrm{tu}\end{array}$ & VG: 1062 \\
\hline \multicolumn{5}{|c|}{ 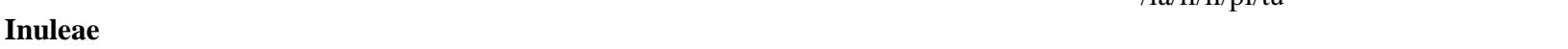 } \\
\hline Achyrocline alata (Kunth) DC. & & $3000-4000$ & $\begin{array}{l}\text { am/an/ap/ar/ay/ca/cu/h } \\
\mathrm{u} / \mathrm{ju} / \mathrm{li} / \mathrm{la} / \mathrm{ll} / \mathrm{mo} / \mathrm{pa} / \mathrm{pi} / \mathrm{pu} \\
/ \mathrm{sm} / \mathrm{ta}\end{array}$ & VG: 7902 \\
\hline Achyrocline ramosissima Britton ex Rusby ${ }^{1}$ & & $2200-4100$ & $\begin{array}{l}\text { an/ar/ay/cu/hv/ju/li } / \mathrm{mo} / \\
\mathrm{pu} / \mathrm{ta}\end{array}$ & VG: 8046 \\
\hline Cuatrecasasiella isernii (Cuatrec.) H. Rob. & & $4000-4700$ & an/hu/ju/li/ll & VG: 7666 \\
\hline Facelis lasiocarpa (Griseb.) Cabrera & & $2600-3600$ & an/ay/cu/hv/ju/li/ll & VG: 4721 \\
\hline Facelis plumosa (Wedd.) Sch.Bip. & & $2700-4000$ & an/ju/li/ll/mo/pu/ta & VG: 7756 \\
\hline Gamochaeta americana (Mill.) Wedd. & & $300-4000$ & $\begin{array}{l}\text { am/an/ap/ay/ca/cu/hu/h } \\
\text { v/ju/la/li/mo/pa }\end{array}$ & VG: 1040 \\
\hline Gamochaeta humilis Wedd. & & $3500-4600$ & an/ay/ju/li/mo/pu & VG: 5798 \\
\hline Gamochaeta purpurea (L.) Cabrera & & $300-3900$ & $\begin{array}{l}\text { an/ap/ar/ca/cu/hu/la/li/l } \\
\text { 1/mo/pu/ta }\end{array}$ & VG: 4885 \\
\hline Gamochaeta spicata Cabrera & & $600-3600$ & $\begin{array}{l}\mathrm{am} / \mathrm{an} / \mathrm{cu} / \mathrm{hu} / \mathrm{hv} / \mathrm{ju} / \mathrm{li} / \mathrm{ll} / \\
\mathrm{mo}\end{array}$ & IM: 81 \\
\hline Loricaria thuyoides (Lam.) Sch.Bip. & & $3500-4500$ & $\begin{array}{l}\text { am/ay/cu/hu/ju/li/li/pa/ } \\
\text { sm }\end{array}$ & VG: 688 \\
\hline Loricaria thyrsoidea (Cuatrec.) M.O. Dillon \& Sagást. & & $3900-4000$ & $\mathrm{cu} / \mathrm{ju} / \mathrm{li}$ & VG: 5526 \\
\hline Mniodes kunthiana (DC.) Freire, Chem, Anderb. \& Urtubey & & $3600-4500$ & an/hu/hv/ju/li/mo/pu/ta & VG: 5543 \\
\hline Mniodes pickeringii (A. Gray) M.O Dillon \& Sagást. & & $3000-4000$ & $\mathrm{cu} / \mathrm{ju} / \mathrm{li} / \mathrm{ll} / \mathrm{ta}$ & VG: 7034 \\
\hline $\begin{array}{l}\text { Mniodes piptolepis (Wedd.) Freire, Chem, Anderb. \& } \\
\text { Urtubey }\end{array}$ & & $3000-4500$ & $\begin{array}{l}\text { an } / \mathrm{ar} / \mathrm{ca} / \mathrm{cu} / \mathrm{ju} / \mathrm{li} / \mathrm{ll} / \mathrm{mo} / \mathrm{p} \\
\mathrm{u}\end{array}$ & VG: 5791 \\
\hline Mniodes pulvinulata Cuatrec. & & $4000-4800$ & an/ay/ju/li/mo/ta & VG: 7004 \\
\hline Pluchea chingoyo (Kunth) DC. & & $5-1000$ & an/ar/ic/la/li/ll/pi/ta & VG: 5576 \\
\hline
\end{tabular}


Tabla 1. Lista de Especies colectadas en la cuenca del rio Chillón. Las abreviaturas departamentales siguen a Brako \& Zarucchi (1993).

TRIBU: ESPECIE
Pseudognaphalium cheiranthifolium (Lam.) Hil. \& B.L. Burtt
Pseudognaphalium dombeyanum (D.C) Anderb.
Pseudognaphalium gaudichaudianium (DC.) Anderb.
Pseudognaphalium lacteum (Meyen \& Walp.) Anderb.
Pseudognaphalium psilophyllum (Meyen \& Walp.) Anderb.
Pseudognaphalium viravira (Molina) Anderb. ${ }^{1}$
Stuckertiella capitata (Wedd.) Beauverd
Tessaria integrifolia Ruiz \& Pav.

\section{Lactuceae}

Hieracium leptocephalium Benth.

Hieracium peruanum E.M.Fries*

Hypochaeris chillensis (Kunth) Britton

Hypochaeris echegarayi Hieron.

Hypochaeris eriolaena (Sch.Bip.) Reiche

Hypochaeris meyeniana (Walp.) Griseb.

Hypochaeris sessiliflora Kunth

Hypochaeris taraxacoides Ball.

Picrosia longifolia D. Don

Sonchus asper (L.) Hill

Sonchus oleraceus L.

Taraxacum fernendezianum Dahlst. ex Skottsb.

Taraxacum officinale F.H. Wigg.

\section{Liabeae}

Chionopappus benthamii S.F. Blake*

Munnozia lyrata (A. Gray) H. Rob. \& Brettell*

Paranephelius ovatus Wedd.

Paranephelius uniflorus Poepp.

Philoglossa peruviana DC.*

\section{Mutisieae}

Chaetanthera peruviana A. Gray

Jungia axillaris (Lag. ex DC.) Sprenger

Jungia pauciflora Rusby

Jungia schuerae Harling*

Leucheria daucifolia (D. Don.) Crisci

Mutisia acuminata Ruiz \& Pav.

Mutisia hastata Cav.*

Mutisia mathewsii Hook. \& Arn.

Onoseris albicans (D. Don) Ferreyra

Onoseris annua Less.*

Onoseris odorata (D. Don) Hook. \& Arn.

Oriastrum cochlearifolium A. Gray*

Perezia coerulescens Wedd.

Perezia multiflora (Humb. \& Bonpl.) Less.

\begin{tabular}{|c|c|c|}
\hline $\begin{array}{c}\text { Rango } \\
\text { Altitudinal }\end{array}$ & $\begin{array}{l}\text { Distribución Perú } \\
\text { (departamentos) }\end{array}$ & Colector \\
\hline $2000-4000$ & $\begin{array}{l}\text { an/ar/ca/cu/hu/hv/li/mo } \\
/ \mathrm{pu}\end{array}$ & VG: 2297 \\
\hline $500-3900$ & $\begin{array}{l}\text { an/ar/ay/ca/cu/hu/hv/ju } \\
/ \mathrm{la} / \mathrm{li} / 1 \mathrm{ll} / \mathrm{mo} / \mathrm{pi} / \mathrm{pu} / \mathrm{sm} / \mathrm{ta}\end{array}$ & VG: 800 \\
\hline $2000-4000$ & an/ar/ju/li/ & VG: 958 \\
\hline $3400-4800$ & an/ar/ay/hu/li/ta/pu & VG: 5815 \\
\hline $3400-4100$ & an/ju/li & IM : 210 \\
\hline $3300-3900$ & $\begin{array}{l}\text { an/ay/ca/cu/hv/ju/li/mo } \\
/ \mathrm{pu} / \mathrm{ta}\end{array}$ & VG: 1458 \\
\hline $3900-4000$ & $\mathrm{ap} / \mathrm{ca} / \mathrm{cu} / \mathrm{hv} / \mathrm{ju} / \mathrm{li} / \mathrm{ta}$ & VG: 7981 \\
\hline $100-3200$ & $\begin{array}{l}\mathrm{am} / \mathrm{an} / \mathrm{ar} / \mathrm{ca} / \mathrm{cu} / \mathrm{hu} / \mathrm{hv} / \mathrm{ic} \\
\text { /ju/la/li/ll/lo/md/mo/pa/ } \\
\mathrm{pi} / \mathrm{sm}\end{array}$ & VG: 5640 \\
\hline $2500-3900$ & an/ar/cu/li/ll & VG: 7599 \\
\hline $2000-4000$ & $\begin{array}{l}\text { am/an/ay/ar/ca/cu/hu/h } \\
\text { v/ic/ju/la/ll/li/mo/pa/ta/ }\end{array}$ & VG: 8053 \\
\hline $2500-4100$ & an $/ \mathrm{ar} / \mathrm{ca} / \mathrm{li} / \mathrm{mo} / \mathrm{ta}$ & VG: 5275 \\
\hline $3000-3500$ & $\mathrm{ju} / \mathrm{li} / \mathrm{pa}$ & IM: 202 \\
\hline $4000-4500$ & an/ca/li/ll & IM: 222 \\
\hline $3600-4700$ & $\mathrm{am} / \mathrm{an} / \mathrm{ju} / \mathrm{li} / \mathrm{ll} / \mathrm{mo} / \mathrm{ta}$ & VG: 1252 \\
\hline $3000-3500$ & $\mathrm{am} / \mathrm{sm} / \mathrm{li}$ & GP: 774 \\
\hline $3200-4800$ & $\begin{array}{l}\text { an/ar/ay/cu/hu/ju/li/lli/p } \\
\text { a/pu/sm/ta }\end{array}$ & VG: 2437 \\
\hline $20-3400$ & an $/ \mathrm{ar} / \mathrm{ca} / \mathrm{ic} / \mathrm{ju} / \mathrm{li} / \mathrm{mo} / \mathrm{pa}$ & GP: 5633 \\
\hline $2000-3800$ & $\mathrm{ca} / \mathrm{cu} / \mathrm{li} / \mathrm{pa} / \mathrm{pi}$ & IM: 191 \\
\hline $5-3800$ & $\begin{array}{l}\text { an } / \mathrm{ar} / \mathrm{ca} / \mathrm{cu} / \mathrm{ju} / \mathrm{ic} / \mathrm{li} / \mathrm{ll} / \mathrm{m} \\
\mathrm{o} / \mathrm{pu} / \mathrm{sm} / \mathrm{ta}\end{array}$ & VG: 5237 \\
\hline $1000-4000$ & $\mathrm{am} / \mathrm{an} / \mathrm{li}$ & AC: 10849 \\
\hline $5-3800$ & $\begin{array}{l}\mathrm{am} / \mathrm{an} / \mathrm{ar} / \mathrm{cu} / \mathrm{hu} / \mathrm{hv} / \mathrm{ju} / \mathrm{li} / \\
\mathrm{mo} / \mathrm{pi} / \mathrm{ta}\end{array}$ & VG: 5233 \\
\hline
\end{tabular}

Figura 9

$500-2000$ an/ar/ca/li/ll

VG: 5338

$3300-3700$ am/ap/ar/ay/ca/hu/hv/li VG: 2172

$3000-4600$ an/ap/ay/ca/cu/ju/li/ll/p VG: 733 $\mathrm{a} / \mathrm{pu} /$

Figura $10 \quad 3800-4500$ an/ca/hu/ju/hv/li/ll/pa VG:3 072

$200-900$ an/ar/li/ll/ta $\quad$ VG: 7876

Figura $112800-3900$ an/ar/hv/li/mo/ta $\quad$ VG: 4253

Figura $12 \quad 700-3500$ an/ay/hv/li/ll $\quad$ VG: 4824

$3000-3600 \mathrm{cu} / \mathrm{hu} / \mathrm{pu} / \mathrm{li} \quad$ VG: 7098

$3000-3800$ an/ca/hu/ll/li/ju $\quad$ VG: 1798

3 800-4 800 an/ar/ay/cu/ju/li/mo/pa/ HB: 8896 $\mathrm{pu} / \mathrm{ta}$

$3000-3900$ an/ap/ar/ay/cu/hv/ju/li/l VG: 1796 $1 / \mathrm{mo} / \mathrm{pi} / \mathrm{ta}$

$2400-4000$ an/ar/hu/hv/li/pu ACe: 221

$3400-4400$ an/ap/ay/cu/hv/ju/li/ll VG: 4846

$200-3500$ an/ap/ca/cu/hu/hv/ju/la/ VG: 2624 li/pa/pi

$2200-3400$ an/ay/li $\quad$ GP: 712

$100-3700$ an/ar/ay/ca/cu/ic/la/ll/li VG: 5332 $/ \mathrm{tu}$

$4300-5100$ an/ju/li/hu IM: 235

$3500-4500$ an/ar/ay/cu/hu/hv/ju/li/ $\quad$ VG: 2509 $\mathrm{mo} / \mathrm{pu} /$

$3500-4800$ an/ap/ar/ay/ca/cu/ju/hv/ VG: 2482 $\mathrm{ju} / \mathrm{li} / \mathrm{ll} / \mathrm{pa} / \mathrm{pu} / \mathrm{ta}$ 
Tabla 1. Lista de Especies colectadas en la cuenca del rio Chillón. Las abreviaturas departamentales siguen a Brako \& Zarucchi (1993).

\begin{tabular}{|c|c|c|c|c|}
\hline TRIBU: ESPECIE & & $\begin{array}{c}\text { Rango } \\
\text { Altitudinal }\end{array}$ & $\begin{array}{c}\begin{array}{c}\text { Distribución Perú } \\
\text { (departamentos) }\end{array} \\
\end{array}$ & Colector \\
\hline Perezia pinnatifida (Humb. \& Bonpl.) Wedd. & & $3200-4600$ & $\begin{array}{l}\text { an/ar/ay/cu/hv/ju/li/mo/ } \\
\mathrm{pa} / \mathrm{pu}\end{array}$ & VG: 5230 \\
\hline Perezia pungens (Humb. \& Bonpl.) Less. & & $3000-4600$ & $\begin{array}{l}\mathrm{am} / \mathrm{an} / \mathrm{ca} / \mathrm{cu} / \mathrm{hv} / \mathrm{li} / \mathrm{ll} / \mathrm{mo} \\
/ \mathrm{pa}\end{array}$ & VG: 4124 \\
\hline Polyachyrus sphaerocephalus D. Don & & $1000-3800$ & $\begin{array}{l}\text { an/ar/ay/ic/ju/li/ll/mo/t } \\
\text { a }\end{array}$ & VG: 2311 \\
\hline Proustia cuneifolia D. Don & & $3100-3600$ & ap/ar/ay/cu/li/mo & VG: 1429 \\
\hline Trixis cacalioides (Kunth) D. Don & & $100-2500$ & $\begin{array}{l}\text { am/an/ar/ay/ca/hv/ic/la/ } \\
11 / \mathrm{li} / / \mathrm{mo} / \mathrm{ta}\end{array}$ & VG: 4840 \\
\hline \multicolumn{5}{|l|}{ Senecioneae } \\
\hline Chersodoma antennaria (Wedd.) Cabrera & & $3900-5600$ & an/ay/cu/ju/li/ll/pa/pu & VG: 5414 \\
\hline Chersodoma juanisernii (Cuatrec.) Cuatrec.* & & $3400-4200$ & $\mathrm{ar} / \mathrm{li}$ & GP: 1222 \\
\hline Gynoxys visoensis Cuatrec.* & & $3600-4000$ & li/pa & VG: 1220 \\
\hline Lomanthus albaniae (H.Beltrán) B. Nord. \& Pelser* & & $3000-3400$ & an/ca/li/ll & VG: 4250 \\
\hline Lomanthus calachaquensis (Cabrera) B. Nord.* & & $3600-3900$ & an/hv/li & VG: 7118 \\
\hline Lomanthus cantensis (Cabrera) P. Gonzáles*** & Figura 13 & $\begin{array}{r}2700-3 \\
100\end{array}$ & li & VG: 4924 \\
\hline Lomanthus cerrateae (Cabrera) B. Nord. \& Pelser* & & $3000-3600$ & an/li & VG: 8045 \\
\hline Lomanthus infernalis (Cuatrec.) H. Beltrán* & & $2800-3000$ & an/li & VG: 4429 \\
\hline Lomanthus subcandidus (A. Gray) B. Nord.* & Figura 14 & $3000-3800$ & an/li & VG: 7991 \\
\hline Lomanthus tovarii (Cabrera) B. Nord. \& Pelser* & Figura 15 & $1500-3000$ & ar/ay/li/ & VG: 7452 \\
\hline Lomanthus velardei (Cabrera) B. Nord. \& Pelser* & & $2000-3000$ & hv/li & VG: 7468 \\
\hline Lomanthus yauyensis (Cabrera) B. Nord. \& Pelser* & & $3000-3900$ & an/ar/ca/li & VG: 4120 \\
\hline Misbrookea strigosissima (A. Gray) V. A. Funk & & $3600-4800$ & an/ay/cu/hu/ju/li/ll/mo & VG: 7549 \\
\hline Paracalia jungioides (Hook. \& Arn.) Cuatrec.* & & $2600-3600$ & an/ca/hv/li/ll & VG: 2597 \\
\hline Pentacalia poeppigiana A. Granda*** & & $2600-3600$ & li & VG: 6095 \\
\hline Pseudogynoxys cordifolia (Cass.) Cabrera* & & $100-900$ & $\mathrm{am} / \mathrm{ca} / \mathrm{ll} / \mathrm{li} / \mathrm{la} / \mathrm{ic} / \mathrm{pi}$ & Visto \\
\hline Senecio breviscapus DC. & Figura 16 & $4000-4500$ & $\begin{array}{l}\text { an/ay/cu/hv/hu/ju/li/mo } \\
/ \mathrm{pa} / \mathrm{ta}\end{array}$ & VG: 2278 \\
\hline Senecio calvus Cuatrec.* & & $4000-5600$ & an/li & IM: 167 \\
\hline Senecio candollii Wedd. & & $3700-4500$ & an $/ \mathrm{ar} / \mathrm{cu} / \mathrm{ju} / \mathrm{li} / \mathrm{hv} / \mathrm{pu} /$ & VG: 2494 \\
\hline Senecio canescens (Humb. \& Bonpl.) Cuatrec. & & $3900-5000$ & $\begin{array}{l}\text { an/ap/ca/hu/ju/ll/lii/pu/s } \\
\mathrm{m} / \mathrm{ta} / \mathrm{pa}\end{array}$ & VG: 1775 \\
\hline Senecio casapaltensis Ball* & & $3900-4500$ & an/ju/li & VG: 5790 \\
\hline Senecio collinus DC. & Figura 17 & $3500-4500$ & $\begin{array}{l}\text { an/ca/cu } \\
\mathrm{ju} / \mathrm{hv} / \mathrm{hu} / \mathrm{li} / \mathrm{ll} / \mathrm{pa} / \mathrm{pu}\end{array}$ & VG: 1728 \\
\hline Senecio comosus Cuatrec. & & $3900-4000$ & an/cu/hu/hv/li & VG: 2495 \\
\hline Senecio condimentarius Cabrera* & & $3500-4500$ & an/ap/hu/ju/li/pu & VG: 2542 \\
\hline Senecio culcitioides Sch.Bip. & & $3600-4000$ & $\begin{array}{l}\mathrm{am} / \mathrm{an} / \mathrm{ar} / \mathrm{cu} / \mathrm{hu} / \mathrm{hv} / \mathrm{li} / \mathrm{s} \\
\mathrm{m} / \mathrm{pu}\end{array}$ & VG: 7671 \\
\hline Senecio danai A. Gray* & & $3600-4600$ & an/ap/hv/ju/li/pa & VG: 4480 \\
\hline Senecio evacoides Sch.Bip. & & $4000-4800$ & $\begin{array}{l}\text { an/ar/ay/cu/hv/ju/li/pa/ } \\
\mathrm{pu}\end{array}$ & VG: 6069 \\
\hline Senecio expansus Wedd. & & $3800-4000$ & $\mathrm{hv} / \mathrm{ju} / \mathrm{li} / \mathrm{mo}$ & IM: 206 \\
\hline Senecio gamolepis Cabrera* & & $4000-4500$ & an/ar/ay/hu/hv/ju/li/mo & VG: 4474 \\
\hline Senecio genisianus Cuatrec.* & & $4000-4500$ & an/ay/hv/ju/li & VG: 5544 \\
\hline Senecio gracilipes A. Gray* & Figura 18 & $2500-3900$ & an/li & VG: 2536 \\
\hline Senecio hohenackeri Sch.Bip. ex Wedd. & Figura 19 & $3000-4500$ & $\begin{array}{l}\text { an/ap/ay/cu/hu/hv/ju/li/ } \\
\mathrm{pu}\end{array}$ & VG: 7041 \\
\hline Senecio hyoseridifolius Wedd. & & $3600-4000$ & an/cu/ju/hv/ju/ll/li & WE \\
\hline Senecio modestus Wedd. & Figura 20 & $3900-5000$ & $\begin{array}{l}\text { an/ap/ar/ay/cu/hu/hv/ju } \\
\text { /li/pu/ta }\end{array}$ & VG: 2558 \\
\hline Senecio nivalis (Kunth) Cuatrec. & & $4000-5000$ & an/hv/ju/li & VG: 5190 \\
\hline Senecio nutans Sch.Bip. & & $3800-4500$ & $\begin{array}{l}\text { an/ar/ay/ca/ju/hv/li/pu/t } \\
\text { a }\end{array}$ & VG: 2507 \\
\hline Senecio pflanzii Cuatrec. & & $3600-4800$ & $\mathrm{cu} / \mathrm{hu} / \mathrm{li} / \mathrm{pu}$ & VG: 5548 \\
\hline Senecio pickeringii A. Gray* & & $3500-3900$ & $\mathrm{hv} / \mathrm{ju} / \mathrm{li}$ & WE \\
\hline Senecio pyrenophilus Cuatrec.* & & $3000-3800$ & an/li & VG: 5910 \\
\hline Senecio repens DC. & & $4000-4500$ & an/ca/hu/hv/ju/li/ll/pa & VG: 7110 \\
\hline
\end{tabular}


Tabla 1. Lista de Especies colectadas en la cuenca del rio Chillón. Las abreviaturas departamentales siguen a Brako \& Zarucchi (1993).

\begin{tabular}{|c|c|c|c|c|}
\hline TRIBU: ESPECIE & & $\begin{array}{c}\text { Rango } \\
\text { Altitudinal } \\
\end{array}$ & $\begin{array}{c}\begin{array}{c}\text { Distribución Perú } \\
\text { (departamentos) }\end{array} \\
\end{array}$ & Colector \\
\hline Senecio rhizomatus Rusby & Figura 21 & $3600-4500$ & $\begin{array}{l}\text { an/ar/ap/cu/hv/ju/li/ll/h } \\
\mathrm{u} / \mathrm{pu}\end{array}$ & VG: 5419 \\
\hline Senecio richii A. Gray** & & $3000-3900$ & li & VG: 2084 \\
\hline Senecio rufescens DC. & & $4000-4500$ & $\begin{array}{l}\text { an/ar/ay/cu/hu/hv/ju/li/ } \\
\mathrm{mo} / \mathrm{pa} / \mathrm{pu} / \mathrm{ta}\end{array}$ & VG: 480 \\
\hline Senecio saxipunae Cuatrec. $* * 1$ & & $3900-4300$ & li & SS: 46 \\
\hline Senecio serratifolius (Meyen \& Walp.) Cuatrec. & & $4000-4500$ & $\begin{array}{l}\text { an/ar/ay/cu/hv/li } / \mathrm{mo} / \mathrm{pu} \\
/ \mathrm{ta}\end{array}$ & VG: 2695 \\
\hline Senecio spinosus DC. & & $3600-4500$ & $\begin{array}{l}\text { an/ay/cu/hu/hv/ju/li/mo } \\
/ \mathrm{pa} / \mathrm{pu} / \mathrm{ta}\end{array}$ & VG: 1743 \\
\hline Senecio tephrosioides Turcz. & & $3300-4850$ & an/cu/li/pi/pu/ & IM: 230 \\
\hline Senecio vulgaris $\mathrm{L}$. & & $50-4000$ & $\begin{array}{l}\text { an/ar/ay/ca/cu/hv/ju/li/l } \\
1 / \mathrm{pu}\end{array}$ & VG: 2781 \\
\hline Werneria apiculata Sch.Bip. & & $4000-4800$ & $\begin{array}{l}\text { an/ar/ay/hu/hv/ju/li/mo } \\
/ \mathrm{ta}\end{array}$ & IM: 226 \\
\hline Werneria caespitosa Wedd. & & $3900-5600$ & an/ar/cu/ju/li/pa/pu/ta & VG: 6062 \\
\hline Werneria carnulosa A.Gray*1 & & $4000-4500$ & $\mathrm{ju} / \mathrm{li}$ & HB:8950 \\
\hline Werneria nubigena Kunth & & $3900-5000$ & $\begin{array}{l}\text { an/ar/ca/cu/hu/hv/ju/la/ } \\
\text { li/ll//pa/pi/pu/sm }\end{array}$ & VG: 1237 \\
\hline Werneria orbignyana A. Gray & & $3200-5100$ & an/cu/hv/li & VG: 2499 \\
\hline Werneria pectinata Lingelsh. & & $4300-4800$ & an/ar/ay/hv/ju/li/mo/pu & GP: 1494 \\
\hline Werneria spathulata Wedd. & & $4100-4500$ & $\mathrm{hu} / \mathrm{hv} / \mathrm{ll} / \mathrm{li} / \mathrm{mo} / \mathrm{pu} / \mathrm{ta}$ & VG: 2557 \\
\hline Werneria pygmaea Gillies ex Hook. \& Arn. & & $3300-5600$ & $\begin{array}{l}\text { an/ar/ap/ca/cu/hu/hv/ju } \\
/ \mathrm{li} / \mathrm{ll} / \mathrm{mo} / \mathrm{pa} / \mathrm{pu} / \mathrm{ta}\end{array}$ & VG: 2463 \\
\hline Werneria villosa A. Gray & & $3900-4900$ & $\begin{array}{l}\text { an/ap/ay/ca/cu/hu/hv/ju } \\
/ \mathrm{la} / \mathrm{ll} / \mathrm{li} / \mathrm{pa} / \mathrm{pi} / \mathrm{pu} / \mathrm{sm}\end{array}$ & VG: 4802 \\
\hline Xenophyllum dactylophyllum (Sch.Bip.) V.A. Funk & & $3900-5500$ & $\begin{array}{l}\text { an/ar/ay/cu/hu/hv/ju/li/ } \\
\mathrm{mo} / \mathrm{pa} / \mathrm{pu}\end{array}$ & VG: 6070 \\
\hline Xenophyllum decorum (S.F. Blake) V.A. Funk * & & $4000-4900$ & an/ay/hv/ju/li & VG: 4461 \\
\hline Xenophyllum digitatum (Wedd.) V.A. Funk & & $4000-4900$ & ay/ar/cu/ju/hv/li/mo & GP: 2781 \\
\hline Xenophyllum poposum (Phil.) V.A. Funk & & $4000-5140$ & ar/ay/cu/ju/li/mo/pu/ta & VG: 5540 \\
\hline Xenophyllum staffordiae (Sandwith) V.A. Funk* & Figura 22 & $3900-5000$ & $\mathrm{ar} / \mathrm{hu} / \mathrm{li} / \mathrm{pu}$ & VG: 5194 \\
\hline
\end{tabular}

${ }^{1}$ Registro nuevo para la cuenca.

*Endémico del Perú, ** Endémico de Lima, ***Endémico de Canta

Abreviación de Colectores: AC: Aedo, Carlos. ACe: Acleto, Cesar. GP: Gonzales, Paul. HB: Beltrán. LG: López Guillen. MI: Meza, Irene. RF: Ramón, Ferreyra. SG: Sullivan, Gene. SS: Sánchez, Sergio. VG: Vilcapoma, Graciela. WE: Wilkes Expedition. 


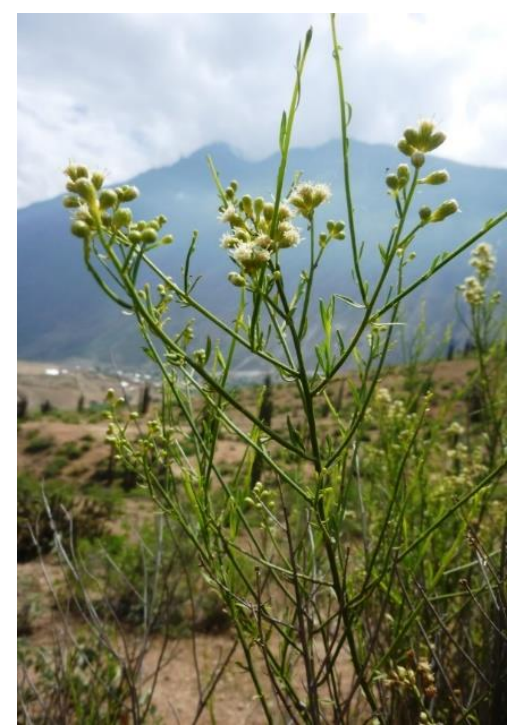

Figura 2.

Baccharis spartea Benth.

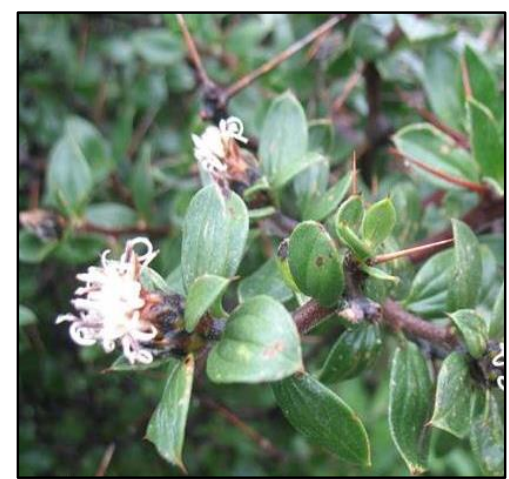

Figura 5.

Dasyphyllum ferox (Wedd.)

Cabrera.

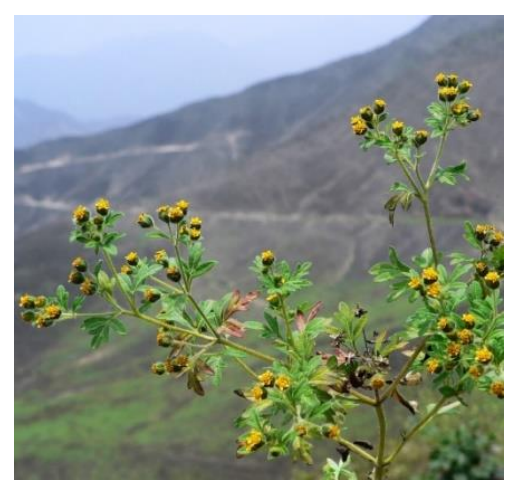

Figura 8.

Villanova oppositifolia (Lag.) S.F. Blake.

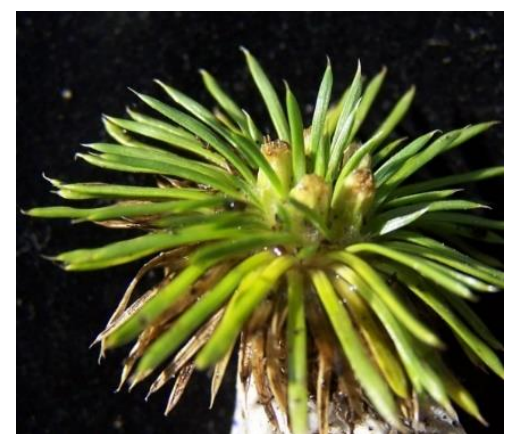

Figura 3.

Novenia acaulis (Wedd. ex Benth.) Freire \& Hellwig.

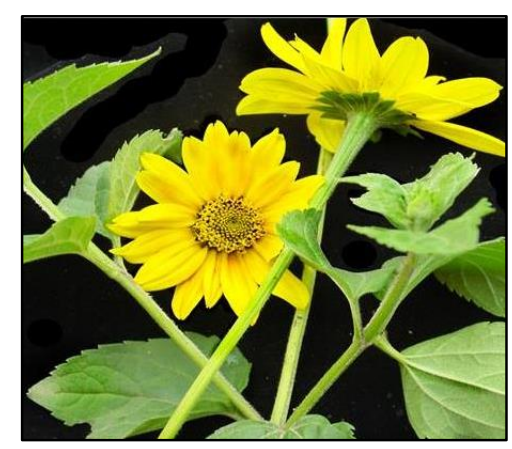

Figura 6.

Heliopsis buphthalmoides

(Jacquin) Dunal.

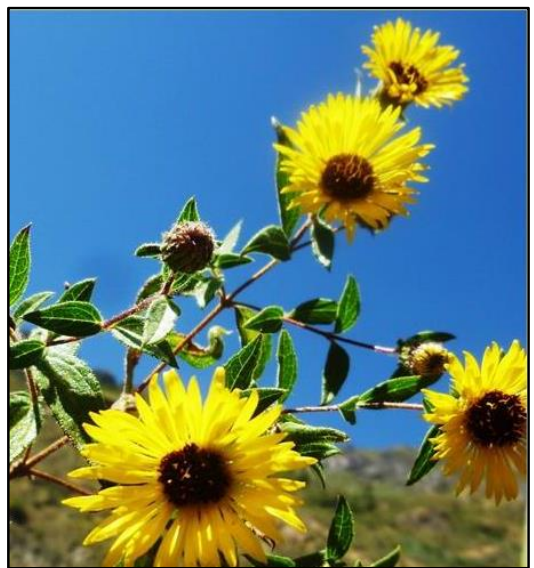

Figura 9.

Chionopappus benthamii S.F. Blake.

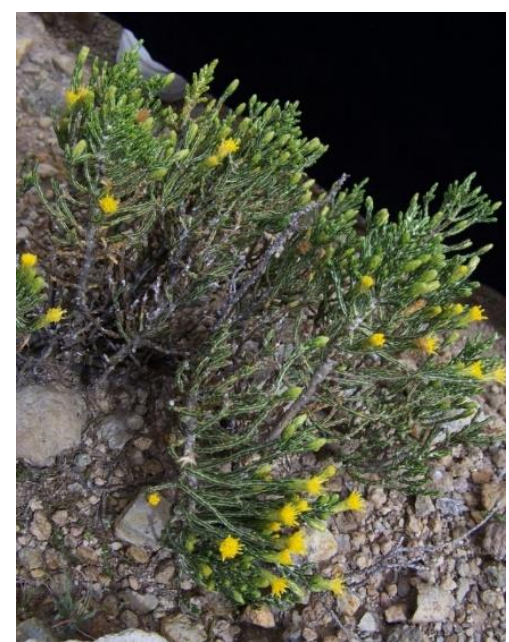

Figura 4.

Parastrephia quadrangularis (Meyen) Cabrera.

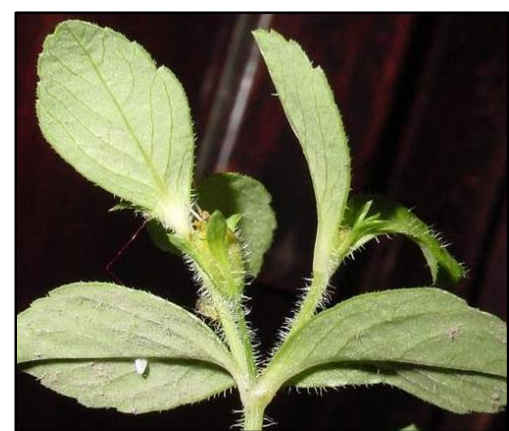

Figura 7.

Heterosperma ovatifolium Cav.

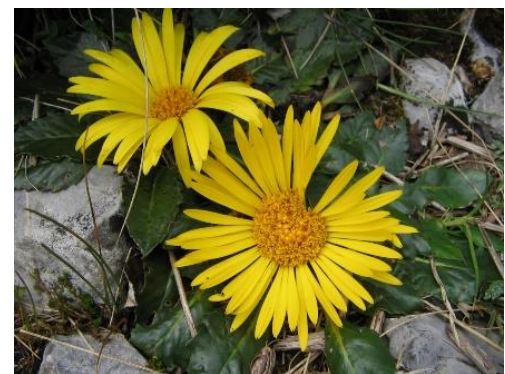

Figura 10.

Paranephelius uniflorus Poepp. \& Endlicher. 


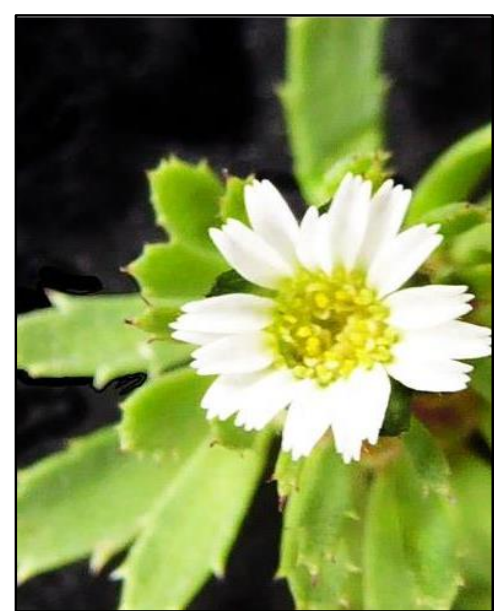

Figura 11.

Chaetanthera peruviana A. Gray.

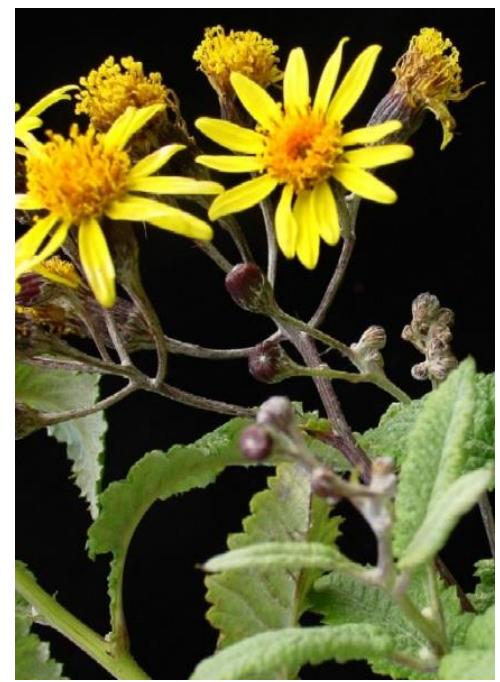

Figura 14.

Lomanthus subcandidus (A. Gray) B. Nord.

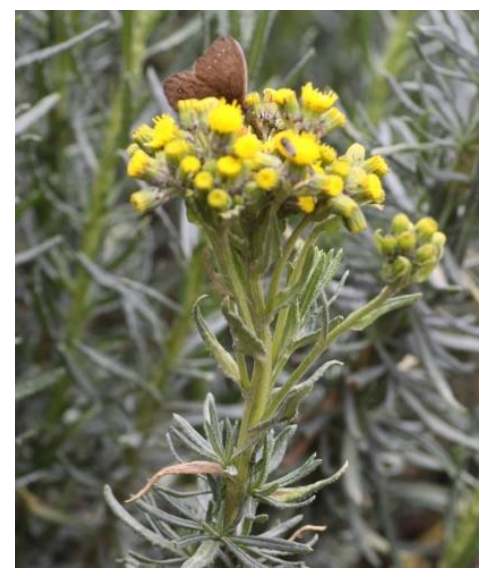

Figura 17.

Senecio collinus DC.

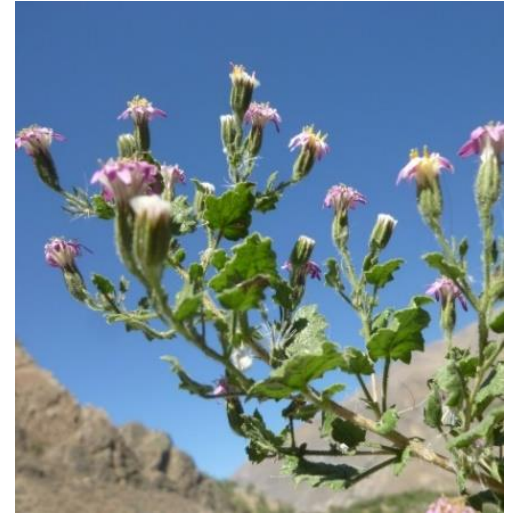

Figura 12.

Jungia axillaris (Lag. ex DC.) Spreng.

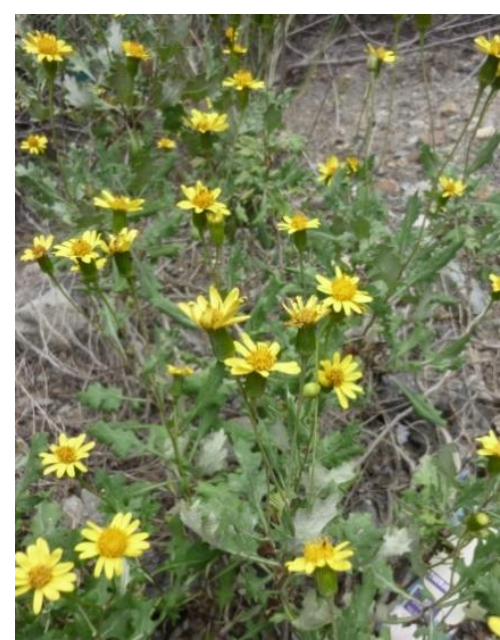

Figura 15.

Lomanthus tovarii (Cabrera) B. Nord. \& Pelser.

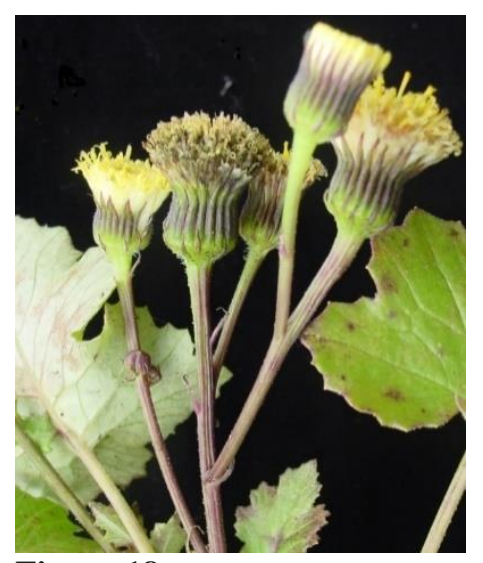

Figura 18.

Senecio gracilipes A. Gray.

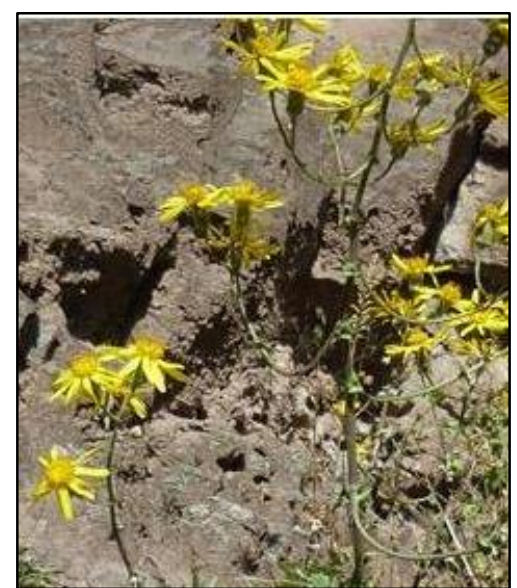

Figura 13.

Lomanthus cantensis (Cabrera) $\mathrm{P}$. Gonzáles.

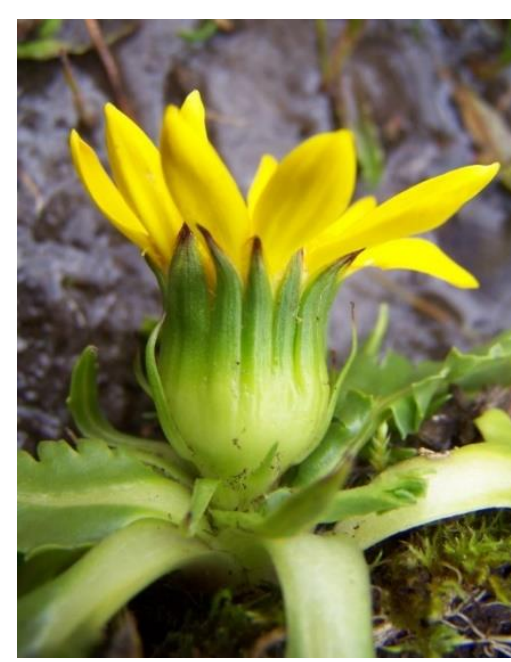

Figura 16.

Senecio breviscapus DC.

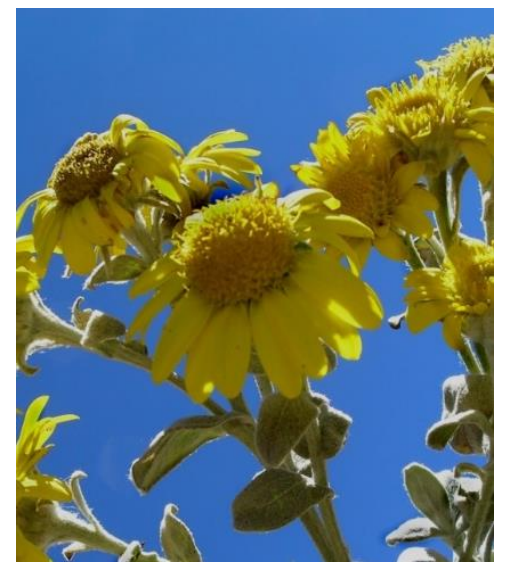

Figura 19.

Senecio hohenackeri Sch.Bip. ex Wedd. 


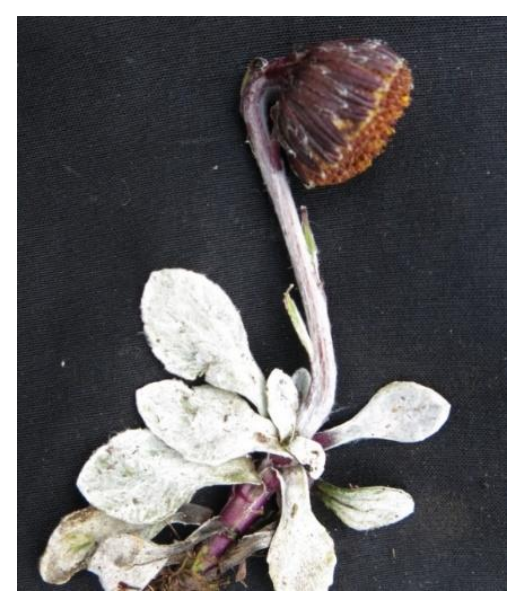

Figura 20.

Senecio modestus Wedd.

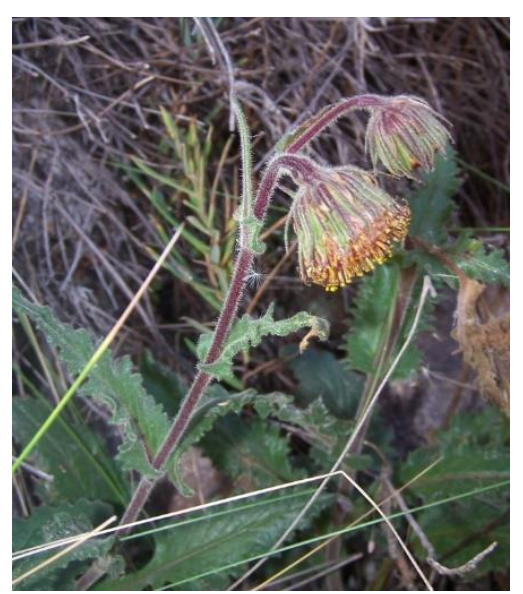

Figura 21.

Senecio rhizomatus Rusby.

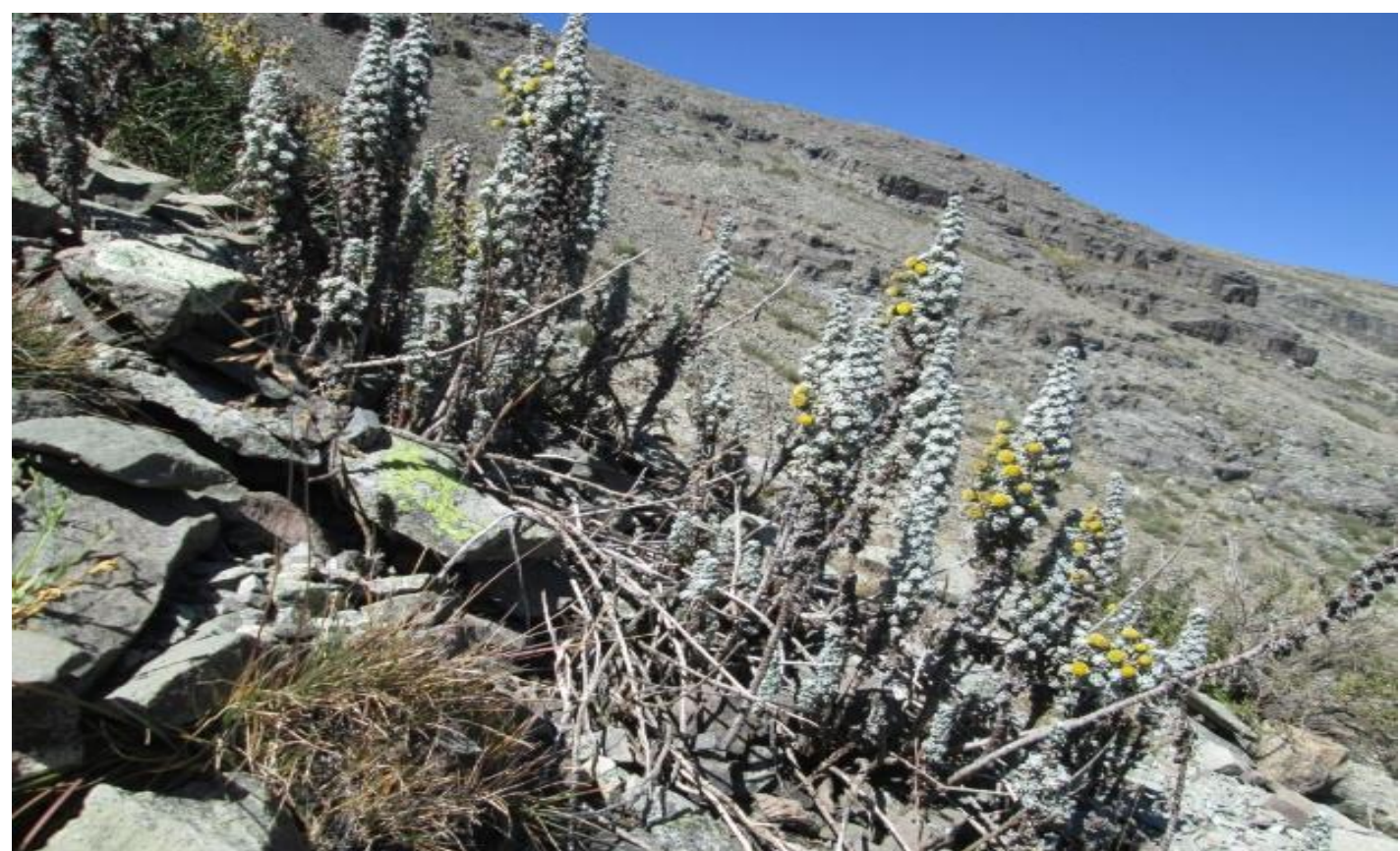

Figura 22. Xenophyllum staffordiae (Sandwith) V.A. Funk.

\footnotetext{
${ }^{1}$ Herbario del Departamento Académico de Biología, Facultad de Ciencias, Universidad Nacional Agraria La Molina. Av. La Universidad s/n, La Molina, Lima, Perú g_vilcapoma@ hotmail.com.

${ }^{2}$ Museo de Historia Natural, Universidad Nacional Mayor de San Marcos. Av. Arenales 1256, Apartado 14-0434, Lima, Perú. hamiltonbeltran@yahoo.com.
} 